\title{
Urinary tract infections: epidemiology, mechanisms of infection and treatment options
}

Ana L. Flores-Mireles*, Jennifer N. Walker*, Michael Caparon and Scott J. Hultgren

Abstract | Urinary tract infections (UTIs) are a severe public health problem and are caused by a range of pathogens, but most commonly by Escherichia coli, Klebsiella pneumoniae, Proteus mirabilis, Enterococcus faecalis and Staphylococcus saprophyticus. High recurrence rates and increasing antimicrobial resistance among uropathogens threaten to greatly increase the economic burden of these infections. In this Review, we discuss how basic science studies are elucidating the molecular details of the crosstalk that occurs at the host-pathogen interface, as well as the consequences of these interactions for the pathophysiology of UTIs. We also describe current efforts to translate this knowledge into new clinical treatments for UTIs.

Pyelonephritis

A kidney infection characterized by cystitis symptoms with additional fever, flank pain, costovertebral-angle tenderness, nausea and vomiting.

Cystitis

An infection of the bladder with accompanying symptoms of dysuria (painful urination), pain (particularly suprapubic), urinary frequency, urinary urgency and haematuria (blood in urine)

Department of Molecular Microbiology and Center for Women's Infectious Disease Research, Washington University School of Medicine, Box 8230, 660 South Euclid Avenue, St. Louis, Missouri 63110-1093, USA

*These authors contributed equally to this work.

Correspondence to S.J.H. e-mail:

hultgren@wusm.wustl.edu doi:10.1038/nrmicro3432

Published online 8 April 2015
Urinary tract infections (UTIs) are some of the most common bacterial infections, affecting 150 million people each year worldwide ${ }^{1}$. In 2007, in the United States alone, there were an estimated 10.5 million office visits for UTI symptoms (constituting $0.9 \%$ of all ambulatory visits) and 2-3 million emergency department visits ${ }^{2-4}$. Currently, the societal costs of these infections, including health care costs and time missed from work, are approximately US $\$ 3.5$ billion per year in the United States alone. UTIs are a significant cause of morbidity in infant boys, older men and females of all ages. Serious sequelae include frequent recurrences, pyelonephritis with sepsis, renal damage in young children, pre-term birth and complications caused by frequent antimicrobial use, such as high-level antibiotic resistance and Clostridium difficile colitis.

Clinically, UTIs are categorized as uncomplicated or complicated. Uncomplicated UTIs typically affect individuals who are otherwise healthy and have no structural or neurological urinary tract abnormalities ${ }^{5,6}$; these infections are differentiated into lower UTIs (cystitis) and upper UTIs (pyelonephritis) ${ }^{5,7}$. Several risk factors are associated with cystitis, including female gender, a prior UTI, sexual activity, vaginal infection, diabetes, obesity and genetic susceptibility ${ }^{3,7}$. Complicated UTIs are defined as UTIs associated with factors that compromise the urinary tract or host defence, including urinary obstruction, urinary retention caused by neurological disease, immunosuppression, renal failure, renal transplantation, pregnancy and the presence of foreign bodies such as calculi, indwelling catheters or other drainage devices $^{8,9}$. In the United States, $70-80 \%$ of complicated UTIs are attributable to indwelling catheters ${ }^{10}$, accounting for 1 million cases per year ${ }^{4}$. Catheter-associated UTIs (CAUTIs) are associated with increased morbidity and mortality, and are collectively the most common cause of secondary bloodstream infections. Risk factors for developing a CAUTI include prolonged catheterization, female gender, older age and diabetes ${ }^{11}$.

UTIs are caused by both Gram-negative and Grampositive bacteria, as well as by certain fungi (FIG. 1). The most common causative agent for both uncomplicated and complicated UTIs is uropathogenic Escherichia coli (UPEC). For the agents involved in uncomplicated UTIs, UPEC is followed in prevalence by Klebsiella pneumoniae, Staphylococcus saprophyticus, Enterococcus faecalis, group B Streptococcus (GBS), Proteus mirabilis, Pseudomonas aeruginosa, Staphylococcus aureus and Candida spp. ${ }^{3,6,12,13}$ (FIG. 1). For complicated UTIs, the order of prevalence for causative agents, following UPEC as most common, is Enterococcus spp., K. pneumoniae, Candida spp., S. aureus, $P$. mirabilis, $P$. aeruginosa and GBS $^{9,14-16}$ (FIG. 1).

Patients suffering from a symptomatic UTI are commonly treated with antibiotics; these treatments can result in long-term alteration of the normal microbiota of the vagina and gastrointestinal tract and in the development of multidrug-resistant microorganisms ${ }^{17}$. The availability of niches that are no longer filled by the altered microbiota can increase the risk of colonization with multidrug-resistant uropathogens. Importantly, the 'golden era' of antibiotics is waning, and the need 
Uncomplicated UTI

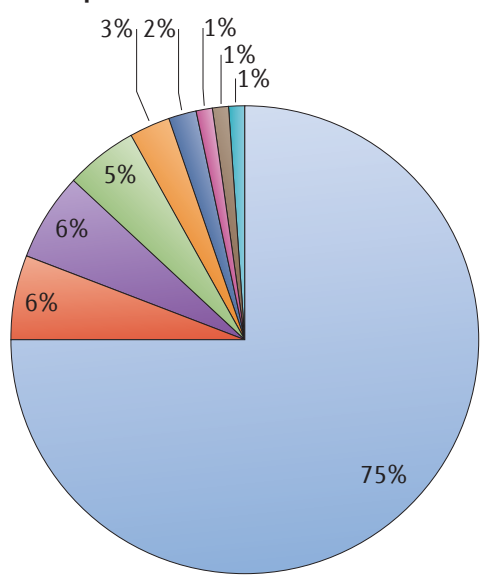

Risk factors

- Female gender

- Older age

- Younger age

\section{Complicated UTI}

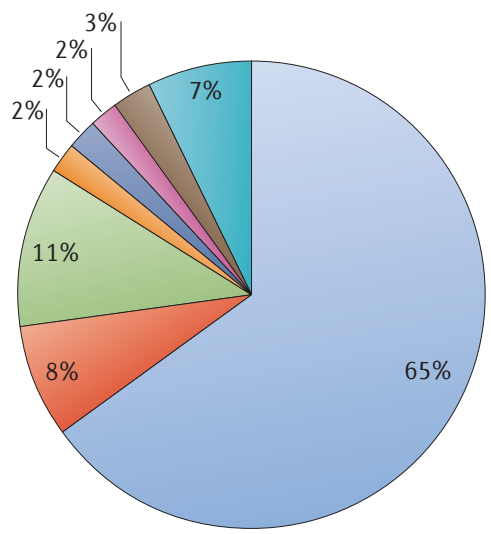

Risk factors

- Indwelling catheters

- Immunosuppression

- Urinary tract abnormalities

- Antibiotic exposure

Figure 1 | Epidemiology of urinary tract infections. Urinary tract infections (UTIs) are caused by a wide range of pathogens, including Gram-negative and Gram-positive bacteria, as well as fungi. Uncomplicated UTIs typically affect women, children and elderly patients who are otherwise healthy. Complicated UTIs are usually associated with indwelling catheters, urinary tract abnormalities, immunosuppression or exposure to antibiotics. The most common causative agent for both uncomplicated and complicated UTIs is uropathogenic Escherichia coli (UPEC). For uncomplicated UTIs, other causative agents are (in order of prevalence) Klebsiella pneumoniae, Staphylococcus saprophyticus, Enterococcus faecalis, group B Streptococcus (GBS), Proteus mirabilis, Pseudomonas aeruginosa, Staphylococcus aureus and Candida spp. For complicated UTIs, the other causative agents are (in order of prevalence) Enterococcus spp., K. pneumoniae, Candida spp., S. aureus, P. mirabilis, P. aeruginosa and GBS.

for rationally designed and alternative treatments is therefore increasing. Recent studies have used RNA sequencing to directly analyse uropathogens from the urine of women experiencing symptomatic UTIs. These studies, together with basic science and improved animal models, have been crucial in enabling us to understand the molecular details of how uropathogens adhere, colonize and adapt to the nutritionally limited bladder environment; evade immune surveillance; and persist and disseminate in the urinary tract. These studies have therefore revealed key virulence factors that can be targeted to prevent and counteract the pathogenic mechanisms that are important in UTIs ${ }^{7,17,18}$. In this Review, we discuss the molecular mechanisms of pathogenesis during bladder and kidney infection, comparing and contrasting the virulence factors used by the major uropathogens UPEC, K. pneumoniae, P. mirabilis, E. faecalis and $P$. aeruginosa. Furthermore, we discuss current antibiotic treatments, antibiotic resistance mechanisms, new combination therapies and future therapeutic interventions that use vaccines and small molecules to target virulence factors.

\section{Adherence and colonization}

Adherence is a key event initiating each step in UTI pathogenesis. A UTI typically starts with periurethral contamination by a uropathogen residing in the gut, followed by colonization of the urethra and subsequent migration of the pathogen to the bladder, an event that requires appendages such as flagella and pili (FIG. 2). In the bladder, the consequences of complex host-pathogen interactions ultimately determine whether uropathogens are successful in colonization or eliminated.

Multiple bacterial adhesins recognize receptors on the bladder epithelium (also known as the uroepithelium) and mediate colonization (TABLE 1). Uropathogens such as UPEC survive by invading the bladder epithelium, producing toxins and proteases to release nutrients from the host cells, and synthesizing siderophores to obtain iron (FIG. 2; TABLE 1). By multiplying and overcoming host immune surveillance, the uropathogens can subsequently ascend to the kidneys, again attaching via adhesins or pili to colonize the renal epithelium and then producing tissue-damaging toxins (FIG. 2; TABLE 1). Consequently, the uropathogens are able to cross the tubular epithelial barrier to access the blood stream, initiating bacteraemia.

The uropathogens that cause uncomplicated UTIs, including UPEC, K. pneumoniae and S. saprophyticus, have the ability to bind directly to the bladder epithelium, which is composed of the umbrella cells (also known as superficial facet cells), intermediate cells and basal cells ${ }^{19}$ (TABLE 1). UPEC and K. pneumoniae bind to uroplakins, which are the major protein components of the umbrella cell apical membrane ${ }^{19}$ and which form a crystalline array protecting the mammalian bladder tissue from damaging agents in urine ${ }^{20}$. In addition to uroplakins, $\alpha_{3} \beta_{1}$ integrins, which are expressed at the surface of uroepithelial cells, can also serve as receptors for UPEC ${ }^{21}$. By contrast, complicated UTIs are initiated 


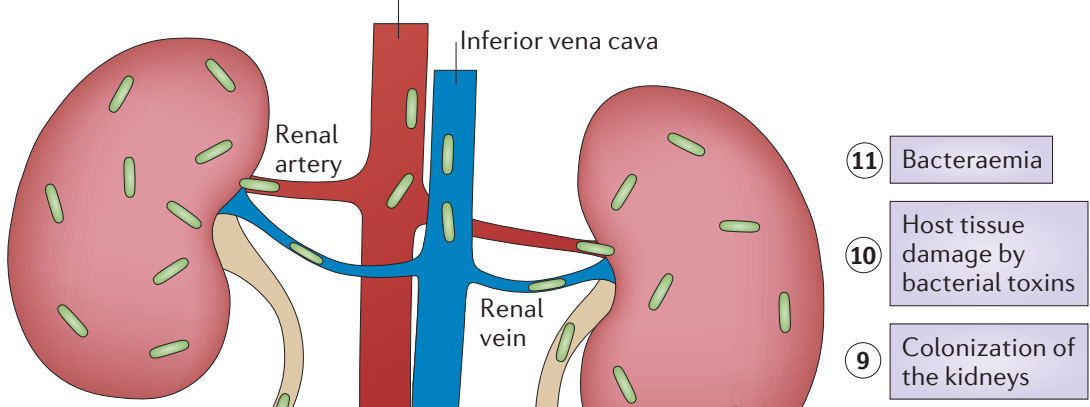

Kidney

$\varnothing$ the kidneys

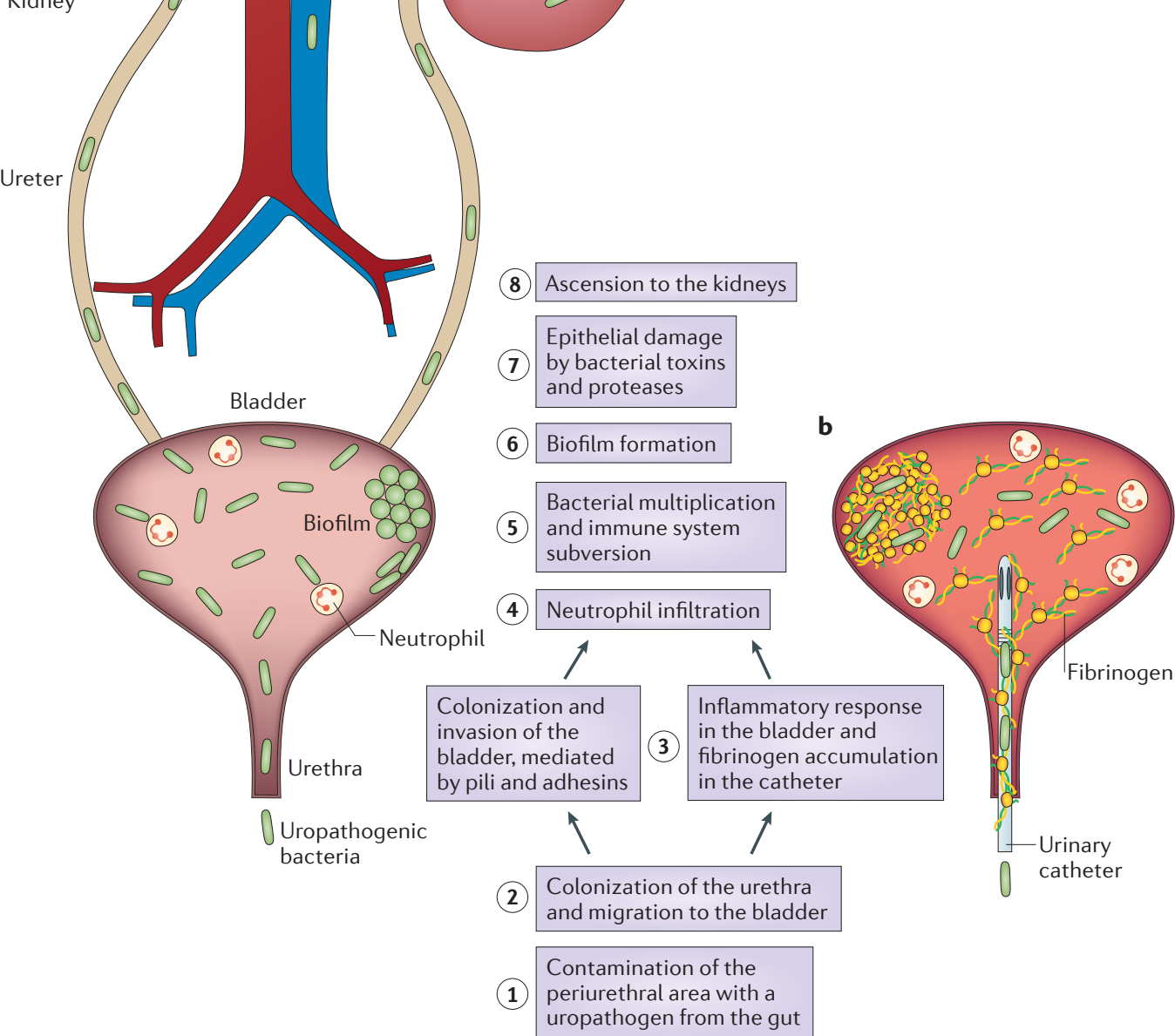

Figure 2| Pathogenesis of urinary tract infections. a |Uncomplicated urinary tract infections (UTIs) begin when uropathogens that reside in the gut contaminate the periurethral area (step 1) and are able to colonize the urethra. Subsequent migration to the bladder (step 2 ) and expression of pili and adhesins results in colonization and invasion of the superficial umbrella cells (step 3). Host inflammatory responses, including neutrophil infiltration (step 4), begin to clear extracellular bacteria. Some bacteria evade the immune system, either through host cell invasion or through morphological changes that result in resistance to neutrophils, and these bacteria undergo multiplication (step 5) and biofilm formation (step 6). These bacteria produce toxins and proteases that induce host cell damage (step 7), releasing essential nutrients that promote bacterial survival and ascension to the kidneys (step 8). Kidney colonization (step 9) results in bacterial toxin production and host tissue damage (step 10). If left untreated, UTIs can ultimately progress to bacteraemia if the pathogen crosses the tubular epithelial barrier in the kidneys (step 11). b| Uropathogens that cause complicated UTIs follow the same initial steps as those described for uncomplicated infections, including periurethral colonization (step 1), progression to the urethra and migration to the bladder (step 2). However, in order for the pathogens to cause infection, the bladder must be compromised. The most common cause of a compromised bladder is catheterization. Owing to the robust immune response induced by catheterization (step 3), fibrinogen accumulates on the catheter, providing an ideal environment for the attachment of uropathogens that express fibrinogen-binding proteins. Infection induces neutrophil infiltration (step 4), but after their initial attachment to the fibrinogen-coated catheters, the bacteria multiply (step 5), form biofilms (step 6), promote epithelial damage (step 7) and can seed infection of the kidneys (steps 8 and 9), where toxin production induces tissue damage (step 10). If left untreated, uropathogens that cause complicated UTIs can also progress to bacteraemia by crossing the tubular epithelial cell barrier (step 11). 
Table 1 | Virulence factors used by the main uropathogens

\begin{tabular}{|c|c|c|c|c|c|c|}
\hline \multirow[t]{2}{*}{ Uropathogen } & \multicolumn{5}{|l|}{ Virulence factors } & \multirow[t]{2}{*}{ Refs } \\
\hline & Adherence & Toxin & Immune evasion & Iron acquisition & Other & \\
\hline UPEC & $\begin{array}{l}\text { - F1C pili } \\
\text { - P pili } \\
\text { - S pili } \\
\text { - Type } 1 \text { pili } \\
\text { - Dr adhesins }\end{array}$ & $\begin{array}{l}\text { - HlyA } \\
\text { - CNF1 }\end{array}$ & $\begin{array}{l}\text { - HlyA } \\
\text { - Capsular antigens } \\
\text { - CNF1 } \\
\text { - Yersiniabactin }\end{array}$ & $\begin{array}{l}\text { - Aerobactin } \\
\text { - Enterobactin } \\
\text { - Salmochelin } \\
\text { - Yersiniabactin }\end{array}$ & $\begin{array}{l}\text { - Antigen43 } \\
\text { - Flagella }\end{array}$ & $\begin{array}{l}\text { 6,33,63,93, } \\
96,141,142\end{array}$ \\
\hline $\begin{array}{l}\text { Klebsiella } \\
\text { pneumoniae }\end{array}$ & $\begin{array}{l}\text { - Type } 1 \text { pili } \\
\text { - Type } 3 \text { pili }\end{array}$ & ND & Capsule & $\begin{array}{l}\text { - Aerobactin } \\
\text { - Enterobactin }\end{array}$ & ND & $49-51,143,144$ \\
\hline Proteus mirabilis & $\begin{array}{l}\text { - MR/P pili } \\
\text { - NAFs } \\
\text { - PMFs } \\
\text { - AipA adhesin } \\
\text { - TaaP adhesin }\end{array}$ & $\begin{array}{l}\text { - Haemolysins } \\
\text { (HpmA and } \\
\text { HlyA) } \\
\text { - Pta }\end{array}$ & $\begin{array}{l}\text { - Capsule } \\
\text { - ZapA }\end{array}$ & $\begin{array}{l}\text { Proteobactin } \\
\text { - Yersiniabactin- } \\
\text { related }\end{array}$ & $\begin{array}{l}\text { - Flagella } \\
\text { - Urease }\end{array}$ & $6,53,97,145$ \\
\hline $\begin{array}{l}\text { Pseudomonas } \\
\text { aeruginosa }\end{array}$ & $\begin{array}{l}\text { - Extracellular DNA } \\
\text { - Exopolysaccharides } \\
\text { (alginate, PEL and } \\
\text { PSL) }\end{array}$ & ND & $\begin{array}{l}\text { - Capsule } \\
\text { - Elastase } \\
\text { - ExoS } \\
\text { - Phospholipase } \\
\text { - Rhamnolipids }\end{array}$ & $\begin{array}{l}\text { - Pyochelin } \\
\text { - Pyoverdin }\end{array}$ & Quorum sensing & $\begin{array}{r}81,86,137 \\
146,147\end{array}$ \\
\hline $\begin{array}{l}\text { Enterococcus } \\
\text { faecalis }\end{array}$ & $\begin{array}{l}\text { - Ebp pili } \\
\text { - Ace adhesin } \\
\text { - Esp adhesin }\end{array}$ & ND & Epa & ND & $\begin{array}{l}\text { - Sortase A } \\
\text { - SigV } \\
\text { - MsrA and MsrB }\end{array}$ & $54,55,61$ \\
\hline $\begin{array}{l}\text { Enterococcus } \\
\text { faecium }\end{array}$ & $\begin{array}{l}\text { - Ebp pili } \\
\text { - Esp adhesin }\end{array}$ & ND & ND & ND & ND & 54 \\
\hline
\end{tabular}

AipA, adhesion and invasion mediated by the Proteus autotransporter; CNF1, cytotoxic necrotizing factor 1; Ebp, endocarditis- and biofilm-associated; Epa, enterococcal polysaccharide antigen; Esp, enterococcal surface protein; ExoS, exoenzyme S; F1C pili, type 1-like immunological group C pili; HlyA, $\alpha$-haemolysin; HpmA, haemolysin; MR/P, mannose-resistant Proteus-like; Msr, methionine sulfoxide reductase; NAF, non-agglutinating fimbria; ND, not determined; PMF,

P. mirabilis-like fimbria; P pili, pyelonephritis-associated pili; Pta, Proteus toxic agglutinin; TaaP, trimeric autoagglutinin autotransporter of Proteus; UPEC, uropathogenic Escherichia coli.

when the bacteria bind to a urinary catheter, a kidney stone or a bladder stone, or when they are retained in the urinary tract by a physical obstruction. Some pathogens (for example, UPEC) can cause both uncomplicated and complicated UTIs. However, others such as P. mirabilis, P. aeruginosa and Enterococcus spp. predominantly cause complicated UTIs (FIG. 2). Subsequently, these uropathogens often form biofilms that are responsible for colonization and persistence ${ }^{22,23}$ (BOX 1).

Chaperone-usher pathway pili. Many uropathogens initiate a UTI using pili that mediate adhesion to host and environmental surfaces, facilitate invasion into the host tissues and promote interbacterial interactions to form biofilms ${ }^{24-27}$. For example, numerous Gram-negative pathogenic bacteria - including E. coli, Klebsiella spp., Proteus spp., Pseudomonas spp., Haemophilus spp., Salmonella spp. and Yersinia spp. ${ }^{16,27-29}$ - express a large, highly conserved family of adhesive fibres called chaperone-usher pathway (CUP) pili ${ }^{25,26}$. CUP pili are assembled by the chaperone-usher molecular machinery ${ }^{24,25}$ and are composed of pilin subunits with incomplete immunoglobulin-like folds that lack the typical carboxy-terminal seventh $\beta$-strand ${ }^{30,31}$. Briefly, in a process termed donor-strand complementation, a dedicated periplasmic chaperone 'donates' a $\beta$-strand to complete the immunoglobulin fold of the subunits, forming a complex with each subunit and ensuring their proper folding and stabilization. The chaperone-subunit complex is then targeted to the usher assembly protein in the outer membrane, where the usher selectively differentiates chaperone-subunit complexes and catalyses the ordered assembly of pili on the cell surface via a mechanism termed donor-strand exchange. During donorstrand exchange, the final folding of a subunit occurs as the donated $\beta$-strand of the chaperone is replaced by an amino-terminal extension on the next incoming subunit ${ }^{32}$. Importantly, understanding the most basic principles of molecular biology - such as how a protein folds into domains that serve as assembly modules for building large supramolecular structures, and how an outer-membrane macromolecular machine (the usher) assembles these structures from individual subunits, which are delivered as chaperone-subunit complexes and then transported in a regulated manner across a biological membrane - has led to the development of antivirulence compounds that block CUP pilus assembly or function and that result in the dysregulation of virulence factors. These compounds have the potential for broadspectrum activity against numerous Gram-negative bacteria (see below).

Uropathogenic Escherichia coli. Thirty-eight distinct CUP pilus operons have been identified in E. coli genomes, and a single UPEC strain can encode more than 12 different CUP pili ${ }^{25}$. However, the distribution of 


\section{Box 1 | Biofilms and morphological plasticity}

Uropathogens use different mechanisms for survival in response to stresses in the bladder such as starvation and immune responses. By forming biofilms and undergoing morphological changes, uropathogens can persist and cause recurrent infections ${ }^{40,129,130}$.

\section{Biofilm formation}

Extracellular DNA (eDNA), exopolysaccharides called extracellular polymeric substances, pili, flagella and other adhesive fibres create a scaffold to form a multicellular bacterial community that is protected from immune responses, antimicrobial agents and other stresses ${ }^{40}$. The antimicrobial recalcitrance of uropathogens increases on biofilm maturation, as the biofilm provides a physical barrier to antibiotic entry. Therefore, understanding species-specific biofilm formation and dispersal mechanisms is crucial for the development of novel therapies that prevent colonization, such as biofilm inhibitors, anti-adhesive molecules and molecules that induce bacterial dispersion.

Uropathogenic Escherichia coli (UPEC) forms biofilm-like intracellular bacterial communities (IBCs) that protect their members from neutrophils, antibiotics and other stresses ${ }^{38}$ (FIG. 3). Type 1 pili, antigen 43 and adhesive surface fibres called curli induce biofilm formation by mediating interbacterial interactions and attachment to surfaces. Transcription of antigen 43 is regulated by oxidative stress regulator (OxyR; also known as hydrogen peroxide-inducible genes activator) ${ }^{131}$, whereas type 1 pilus and curli fibre genes are regulated by polymyxin-resistant protein B (PrmB; also known as BasS) on iron sensing ${ }^{3}$, leading to phosphorylation of polymyxin-resistant protein A (PmrA; also known as BasR) and quorum sensing regulator $B(Q s e B)^{131}$. UPEC biofilm formation on catheters is dependent on type 1 pili $^{35}$.

Proteus mirabilis produces urease, which hydrolyses urea to carbon dioxide and ammonia. This increases the urine $\mathrm{pH}$ and generates calcium crystals and magnesium ammonium phosphate precipitates, which are incorporated into polysaccharide capsules, forming crystalline biofilms on the catheter (FIG. 4). The phosphotransferase regulator of swarming behaviour (RsbA) upregulates polysaccharide expression, represses swarming ${ }^{23}$ and enhances biofilm formation. Mannose-resistant Proteus-like (MR/P) pili intimately associate with the crystal layers, promoting biofilm formation. Oxygen limitation in the biofilm activates the expression of MR/P pili by inducing the recombinase Mrpl to reorient the promoter of the pilus genes. Similarly, expression of the fimbrial operon regulator MrpJ leads to decreased motility, promoting biofilm formation ${ }^{53,132}$.

Pseudomonas aeruginosa has the ability to form biofilms on catheters and damaged bladder tissue ${ }^{82}$ through several mechanisms, including quorum sensing autoinducers that bind to the transcriptional regulators LasR (which regulates elastase (LasB) expression) and RhIR (which regulates the synthesis of rhamnolipids). Quorum sensing induces the production of eDNA, rhamnolipids, lectins, elastases and toxins. The amphiphilic rhamnolipids allow microcolony formation by changing the hydrophobicity of the P. aeruginosa surface ${ }^{133}$. Biofilm maturation is promoted by lectin adhesins, which are important for bacterial cell-cell interactions ${ }^{134}$. The production of alginates and extracellular polymeric substances is activated when cyclic di-GMP binds to the transcriptional regulators alginate biosynthesis 44 (Alg44) and pellicle formation regulator D (PelD) $)^{135}$. Small RNAs from the regulator of secondary metabolites ( $\left.r s m\right)$ family, such as $r s m Z$ and $r s m Y$, regulate exopolysaccharide production by reducing the availability of RsmA, which is the transcriptional repressor for exopolysaccharide-encoding genes ${ }^{81,136,137}$.

\section{Morphological changes}

Uropathogens also adopt morphological changes, such as filamentation, to circumvent the host immune system ${ }^{130,138}$. During IBC maturation, expression of suppressor of lon (SulA) inhibits FtsZ polymerization in a subpopulation of UPEC, blocking septation ring formation and cell division ${ }^{138}$. When the resulting filamentous bacterial cells emerge from epithelial cells, they are resistant to killing by neutrophils and can colonize other naive uroepithelial cells and re-enter the IBC cycle ${ }^{129,138}$ (FIG. 3). Alternatively, during colonization by P. mirabilis, the bacteria adopt a filamentous morphology as a result of the sensor activities of flagella on contact with a urinary catheter. Contact creates a torsional change in the outer membrane, and this is sensed by upregulator of the flagellar master operon (Umo) proteins, which induce the expression of flagella to produce the highly flagellated cells that are required for swarming during a UTI6,23,53,139 (FIC. 4).

CUP operons is not uniform across different UPEC isolates; some operons are found ubiquitously in UPEC, whereas others are present in only a handful of strains. The multitude of CUP pili encoded by UPEC are tipped with different adhesins, some of which are known to mediate distinct tropisms in the lower and upper urinary tract by recognizing receptors with stereochemical specificity, notably in the bladder or kidney epithelium ${ }^{33}$.

Type 1 pili and pyelonephritis-associated (P) pili are the better characterized CUP pili. Type 1 pili are essential for colonization, invasion and persistence of UPEC in the mouse bladder ${ }^{34}$ (FIG. 3). Type 1 pili are tipped with the adhesin $\mathrm{FimH}^{7}$, which recognizes mannosylated uroplakins and $\alpha_{1} \beta_{3}$ integrins with stereochemical specificity ${ }^{21,35}$ to initiate colonization and invasion into umbrella cells $^{7,21}$. Type 1 pili binding to these cells triggers a signal transduction cascade that activates Rho GTPases, such as those from the Rac family, to cause actin rearrangement and internalization of UPEC by a zippering mechanism consisting of a plasma membrane sheathe that engulfs the bacterium ${ }^{36}$ (FIG. 3). Invasion allows UPEC to subvert certain host defences and become recalcitrant to antibiotic treatments. However, an innate defence expulsion mechanism defends the uroepithelium from UPEC invasion; this expulsion mechanism depends on Toll-like receptor 4 (TLR4) expression by uroepithelial cells. Lipopolysaccharide (LPS)-mediated activation of TLR4 stimulates adenylyl cyclase 3 (AC3) to produce cyclic AMP, which induces the exocytosis of vesicular UPEC into the apical plasma membrane of the umbrella cells $^{37}$ (FIG. 3). Importantly, by escaping into the cytoplasm (through an unknown mechanism), UPEC can subvert the expulsion pathway and rapidly multiply, forming transient biofilm-like intracellular bacterial 
a
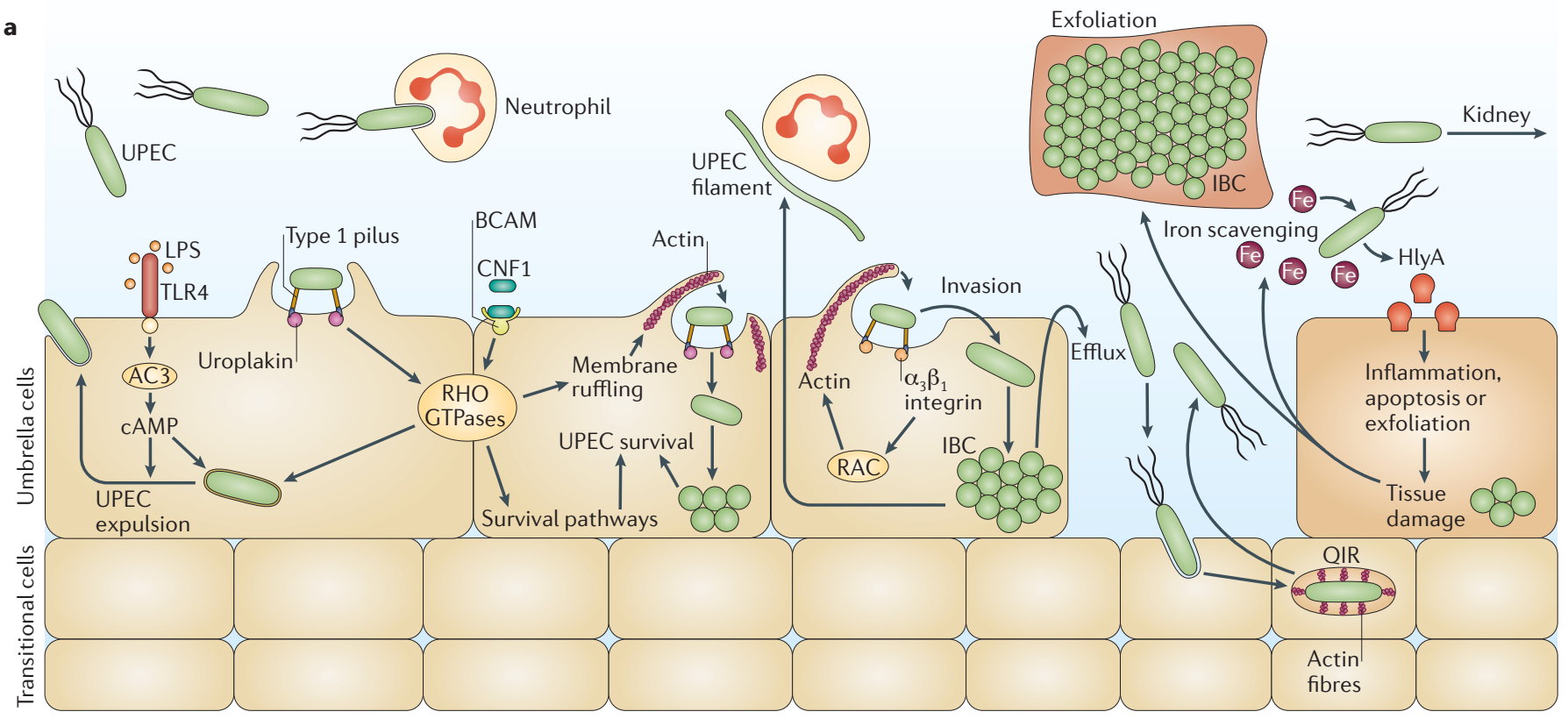

Figure 3 | Virulence factors of uropathogenic Escherichia coli that contribute to urinary tract infections. a | In the bladder, uropathogenic Escherichia coli (UPEC) expression of type 1 pili is essential for colonization, invasion and persistence. The type 1 pilus adhesin, FimH, binds mannosylated uroplakins and integrins that coat the surface of umbrella cells. Uroplakin binding by FimH induces actin rearrangement and bacterial internalization via unknown mechanisms. FimH- $\alpha_{3} \beta_{1}$ integrin interactions induce actin rearrangement via activation of RHO-family GTPases (such as RAC proteins), resulting in bacterial invasion. Inside the host cell, UPEC can subvert host defences and resist antibiotic treatment. However, lipopolysaccharide (LPS) released by UPEC is sensed by Toll-like receptor 4 (TLR4), which induces cyclic AMP (cAMP) production via adenylyl cyclase 3 (AC3) activation, resulting in exocytosis of vesicular UPEC across the apical plasma membrane. UPEC

b

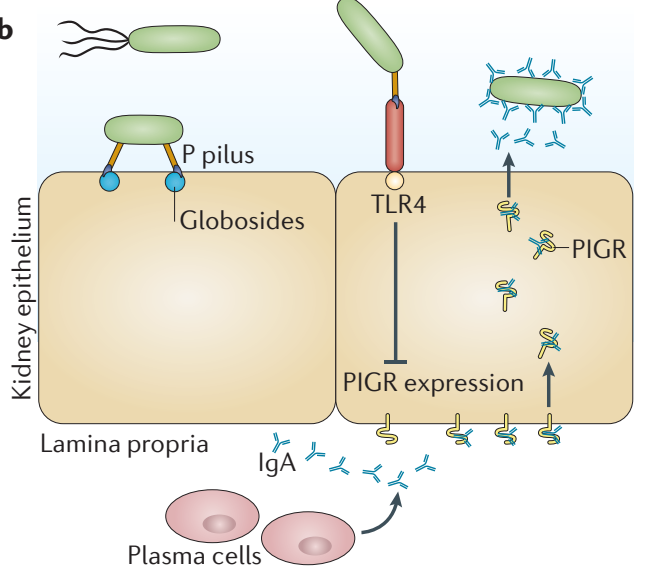
subverts this innate defence mechanism by escaping into the cytoplasm, where it then multiplies to form intracellular bacterial communities (IBCs). Maturation of IBCs causes bacterial dispersal and allows the invasion of other host cells, which enables UPEC to re-enter the IBC cycle. Alternatively, UPEC can establish quiescent intracellular reservoirs (QIRs) in the underlying transitional cells. QIRs consist of 4-10 non-replicating bacteria within membrane-bound compartments encased in F-actin and can remain viable for months. In addition, UPEC survives within the harsh bladder environment by secreting several factors that are important for nutrient acquisition. The toxin $\alpha$-haemolysin (HlyA) promotes host cell lysis through pore formation, facilitating iron release and nutrient acquisition. The siderophores expressed by UPEC allow the bacterium to scavenge iron and thus promote survival during a urinary tract infection (UTI). HlyA also triggers epithelial exfoliation to promote the spread of UPEC to other hosts following urine expulsion or to expose deeper layers of the uroepithelium for QIRs. Cytotoxic necrotizing factor 1 (CNF1) is also important for host cell remodelling and functions by binding to the receptor basal cell adhesion molecule (BCAM) on host cells to induce constitutive activation of the RHO GTPases RAC1, RHOA and cell division control 42 (CDC42), resulting in actin cytoskeletal rearrangements and membrane ruffling. Activation of RAC1 also induces the host cell anti-apoptotic and pro-survival pathways, preventing apoptosis of colonized epithelial cells and allowing the UPEC population to expand. The extracellular survival of UPEC also requires evasion of the innate immune system by the adoption of a filamentous morphology, which renders the bacterium more resistant to neutrophil killing than their bacillary form. b|UPEC colonization of the kidneys is dependent on expression of pyelonephritis-associated (P) pili, which bind globoside-containing glycolipids lining the renal tissue. The P pilus adhesin, PapG, also interacts with TLR4, reducing the expression of polymeric immunoglobulin receptor (PIGR). This results in impaired immunoglobulin A (lgA) transport across the epithelium, thereby modulating the local secretory antibody immune response and preventing UPEC opsonization and clearance.

communities (IBCs) ${ }^{38,39}$ (BOX 1; FIC. 3). After their maturation, bacteria disperse from the IBC to invade other cells, where the IBC cycle is repeated ${ }^{38-40}$. IBC formation is a common mechanism for clinical UPEC isolates and has been observed in multiple mouse backgrounds and also in exfoliated uroepithelial cells in the urine of patients with acute UTIs but not in the cells in urine from healthy controls ${ }^{41,42}$. The process of invasion and 
IBC formation provides UPEC with the ability to survive stringent bottlenecks in the urinary tract, including TLR4-mediated expulsion, umbrella cell exfoliation, ascension to the kidneys, urination and inflammation $^{7,43}$. UPEC also establishes quiescent intracellular reservoirs (QIRs) in underlying transitional cells, within membrane-bound compartments enmeshed in F-actin (FIG. 3). In contrast to the metabolically active IBCs, QIRs typically contain 4-10 non-replicating bacteria that can remain viable for months and can be re-activated to serve as seeds that initiate a recurrent $\mathrm{UTI}^{7}$. It has been proposed that during uroepithelial turnover, in which the underlying immature cells terminally differentiate into umbrella cells, the redistribution of actin and perhaps other associated signals might trigger UPEC revival from QIRs, releasing the bacteria back into the bladder lumen ${ }^{44}$.

Unlike the mannose-binding adhesin FimH of type 1 pili, the adhesin of P pili, PapG, binds globosides containing glycolipids that are present in the human kidneys ${ }^{33}$ (FIG. 3). In addition, PapG modulates the local secretoryantibody immune response by interacting with TLR4 to reduce polymeric immunoglobulin receptor (PIGR) expression, thus impairing immunoglobulin A transport through the lamina propria and epithelial cells to the kidney lumen ${ }^{45}$ (FIG. 3). By inhibiting immunoglobulin A transport into the urinary space, UPEC evades a key host protective mechanism, allowing the establishment of ascending infection ${ }^{45,46}$.

Importantly, the initial innate host response to UPEC colonization and invasion not only dictates the outcome of the original infection but is also crucial for determining host susceptibility to subsequent infections ${ }^{39}$. An increased susceptibility to recurrent UTIs can occur not because of a deficient host response to UPEC infection, as is commonly accepted, but rather as a result of an unrestrained lymphocyte-dependent innate inflammatory response to acute infection, leading to severe acute injury to the mucosal uroepithelium and potentiating subsequent infections $s^{39}$.

Klebsiella pneumoniae. Similarly to UPEC, K. pneumoniae uses type 1 pili for biofilm formation and bladder colonization ${ }^{47}$ (TABLE 1). Interestingly, although the K. pneumoniae adhesin FimH is highly homologous to UPEC FimH, they have different binding specificities ${ }^{48}$. $K$. pneumoniae FimH-mediated biofilm formation is inhibited by heptyl mannose, as opposed to the methyl mannose-mediated inhibition of UPEC FimH. Moreover, $K$. pneumoniae FimH has a weaker adherence to the bladder than UPEC FimH, resulting in significantly lower $K$. pneumoniae titers in the mouse bladder and fewer IBCs than are seen for UPEC. Despite the relatively poor adhesive properties of $K$. pneumoniae FimH in the urinary tract, it remains an important virulence factor for $K$. pneumoniae during colonization, biofilm formation and persistence in both UTIs and CAUTIs ${ }^{48-50}$. In addition, K. pneumoniae encodes numerous other CUP pili, including type 3 pili, which also play an important part in colonization, biofilm formation and persistence during UTIs and in biofilm formation during CAUTIs ${ }^{35,51,52}$.
Proteus mirabilis. Following initial attachment, P. mirabilis produces mannose-resistant Proteus-like (MR/P) pili, which are CUP pili that facilitate biofilm formation and colonization of the bladder and kidneys, and are crucial for catheter-associated biofilm formation ${ }^{6,16,23,53}$ (BOX 1; FIG. 4). Other CUP pili encoded by P. mirabilis include $P$. mirabilis-like fimbriae (PMFs), which are important for bladder and kidney colonization ${ }^{53}$, and non-agglutinating fimbriae (NAFs), which are able to attach to uroepithelial cells in vitro ${ }^{53}$. However, the in vivo mechanistic roles of PMFs, NAFs and their receptors have not yet been established.

In addition to CUP pili, $P$. mirabilis encodes two autotransporters, TaaP (trimeric autoagglutinin autotransporter of Proteus) and AipA (adhesion and invasion mediated by the Proteus autotransporter), which are important for bladder and kidney infection, respectively ${ }^{53}$. AipA can adhere to human bladder and kidney cell lines in vitro but is only required for kidney infection (and not for bladder infection) in mice. Conversely, TaaP is required for bladder infection by $P$. mirabilis in mice. Importantly, both autotransporters bind to extracellular-matrix proteins in vitro: AipA preferentially binds to collagen I, and TaaP to laminin, which might provide an explanation for their different tissue tropisms.

Enterococci. Enterococci encode several adhesion factors, including the collagen adhesin Ace, enterococcal surface protein (Esp), enterococcal polysaccharide antigen (Epa), and endocarditis- and biofilm-associated (Ebp) pili ${ }^{54}$ (TABLE 1). Of these, Ebp pili contribute to CAUTIs $^{54-56}$ and are required for persistence during infection ${ }^{55,56}$. Clinical studies have shown that mechanical stress induced by urinary catheterization produces histological and immunological changes in the bladder, resulting in a robust inflammatory response, exfoliation, oedema, and mucosal lesions of the uroepithelium and kidneys ${ }^{57,58}$. Importantly, a mouse model of CAUTI seems to recapitulate these immunological changes that are induced by urinary catheterization, exhibiting catheter-induced inflammation, severe uroepithelial damage, exfoliation and the onset of bladder wall oedema, which is exacerbated by increased catheterization time ${ }^{59}$. Urinary catheters provide a surface for E. faecalis attachment and biofilm formation, which promotes E. faecalis persistence in the bladder and further dissemination to the kidneys ${ }^{55}$ (FIG. 4). However, E. faecalis is unable to bind to catheter material in vitro and is unable to grow in urine ${ }^{60}$. This apparent paradox was resolved by the finding that urinary catheterization induces fibrinogen release into the bladder as part of the inflammatory response; this fibrinogen subsequently accumulates in the bladder and is deposited on the implanted catheter ${ }^{60}$ (FIGS 2,4). Following fibrinogen deposition, the Ebp pilus adhesin - EbpA, which contains an N-terminal fibrinogen-binding domain - mediates catheter colonization and biofilm formation during CAUTIs caused by E. faecalis ${ }^{60,61}$ (FIG. 4). Furthermore, E. faecalis can use fibrinogen for growth, enhancing biofilm formation on the catheter ${ }^{60}$ (FIG. 4). This resolution of the paradox has 

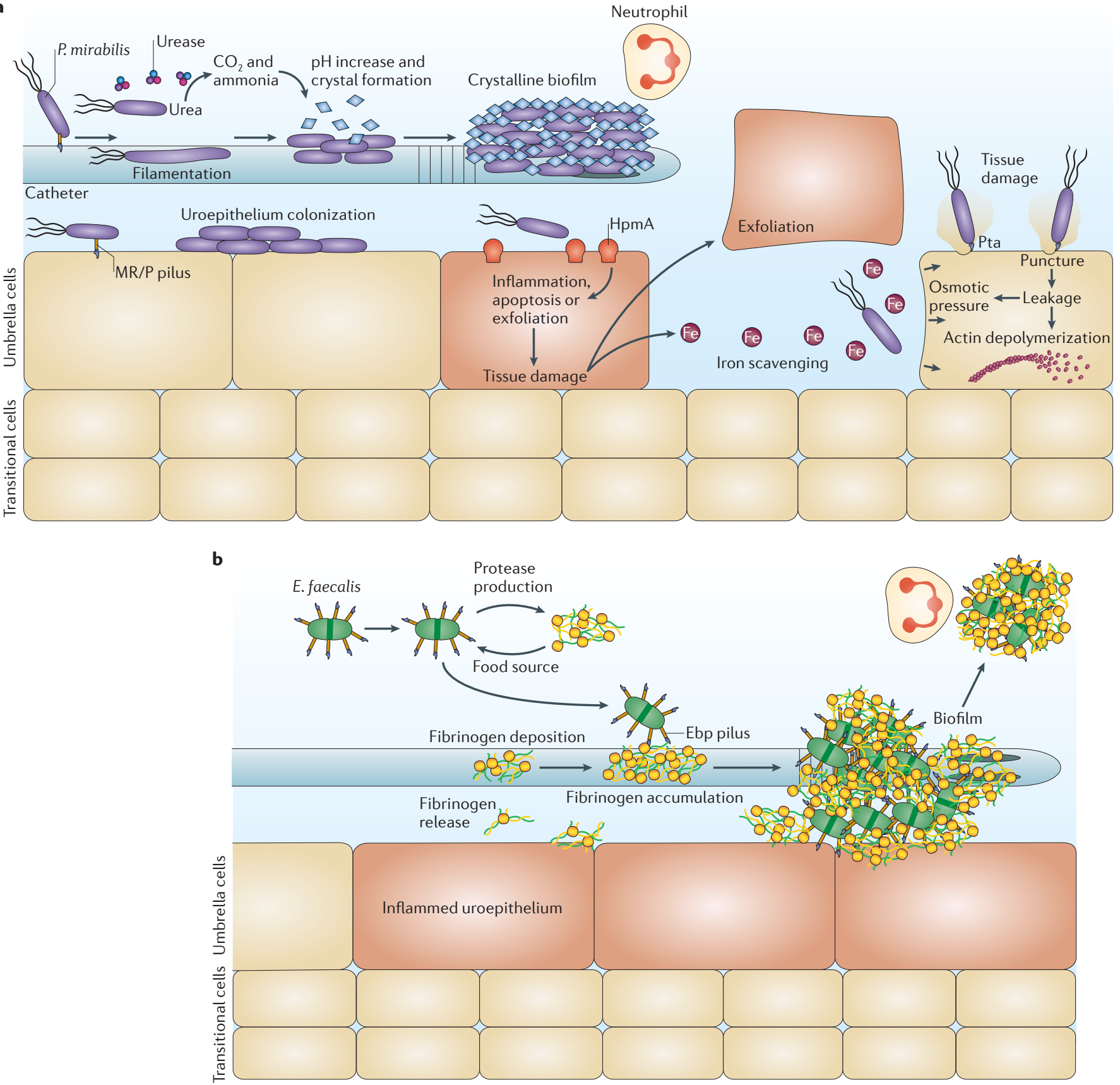

Figure 4 | Mechanisms of pathogenesis during catheter-associated urinary tract infections. a |Catheter-associated urinary tract infections (CAUTIs) mediated by Proteus mirabilis depend on the expression of mannose-resistant Proteus-like (MR/P) pili for initial attachment, and for biofilm formation on the catheter and in the bladder. Subsequent urease production induces the formation of calcium crystals and magnesium ammonium phosphate precipitates in the urine through the hydrolysis of urea to carbon dioxide and ammonia, resulting in a high $\mathrm{pH}$. The production of extracellular polymeric substances by bacteria attached to the catheter traps these crystals, allowing the formation of a crystalline biofilm, which protects the community from the host immune system and from antibiotics. In addition, these structures prevent proper urine drainage, resulting in reflux and promoting the progression to pyelonephritis, septicaemia and shock. Finally, production of the bacterial toxins haemolysin ( $\mathrm{HpmA}$ ) and Proteus toxic agglutinin (Pta) is important for tissue destruction and bacterial dissemination to the kidneys. HpmA induces pore formation by inserting itself into the cell membrane and destabilizing the host cell, causing tissue damage, exfoliation and nutrient release. Pta punctures the host cell membrane, causing cytosol leakage and resulting in osmotic stress and depolymerization of actin filaments, thus compromising the structural integrity of the cell. The release of nutrients via these toxins also allows the bacteria to scavenge iron using siderophores. $\mathbf{b}$ | Enterococcus faecalis pathogenesis during CAUTIs depends on catheter implantation, which results in bladder inflammation and causes fibrinogen release, deposition onto the catheter, and accumulation. E. faecalis takes advantage of the presence of fibrinogen and uses it as a food source through the production of proteases. E. faecalis also binds fibrinogen through the endocarditis- and biofilm-associated (Ebp) pilus, allowing the formation of biofilms that protect the bacteria against the immune system. 
been recapitulated in vitro by the demonstration that E. faecalis attaches to fibrinogen-coated catheters and grows in urine supplemented with fibrinogen ${ }^{60}$.

\section{Other virulence factors}

The bladder environment is limited in nutrients; thus, in order to survive and grow within the urinary tract, uropathogens produce proteases and toxins that damage the host tissue to release nutrients, while also providing a niche for bacterial invasion and dissemination (TABLE 1).

Proteases and toxins. UPEC secretes high concentrations of $\alpha$-haemolysin (HlyA), which oligomerizes and integrates in the cholesterol-rich microdomains in the host cell membrane in a $\mathrm{Ca}^{+}$-dependent manner ${ }^{62,63}$. This results in pore formation in the umbrella cells and promotes their lysis, which facilitates iron and nutrient acquisition by the bacteria (FIG. 3). HlyA also triggers exfoliation, exposing deeper layers of the uroepithelium for colonization and promoting bacterial spread to other hosts following cell expulsion in the urine ${ }^{62-65}$ (FIG. 3). Furthermore, HlyA is highly expressed in IBCs, suggesting that it is important during this stage of infection ${ }^{39,63,66}$.

UPEC also secretes cytotoxic necrotizing factor 1 (CNF1), which affects actin remodelling in the host cell through three small RHO GTPases: RAC1, RHOA and cell division control 42 (CDC42) ${ }^{67,68}$. CNF1 enters the host cell in endocytic vesicles, by binding to the receptor basal cell adhesion molecule (BCAM; also known as $\mathrm{LU})^{69}$, and then constitutively activates RHO GTPases via deamination of a glutamine residue; this causes actin cytoskeletal rearrangements and membrane ruffling, leading to increased levels of bacterial internalization $^{67,70}$. In addition, the activation of RAC1-GTP induces the host cell anti-apoptotic and pro-survival pathways (through the interaction of phosphoinositide 3-kinase (PI3K), AKT (also known as PKB) and nuclear factor- $\kappa \mathrm{B}(\mathrm{NF}-\kappa \mathrm{B})$ ); this prevents apoptosis of the colonized uroepithelium, thus facilitating UPEC survival and protecting the niche ${ }^{67,71}$ (FIG. 3).

P. mirabilis produce two toxins, haemolysin (HpmA) and Proteus toxic agglutinin (Pta), which are implicated in tissue damage and dissemination to the kidneys, initiating acute pyelonephritis ${ }^{16,72}$. HpmA is a $\mathrm{Ca}^{+}$-dependent pore-forming cytolysin that destabilizes the host cell by inserting itself into the cell membrane and causing a $\mathrm{Na}^{+}$efflux ${ }^{16}$ (FIG. 4). By contrast, the surface-associated cytotoxic protease Pta is functional only in an alkaline $\mathrm{pH}$, such as that induced by the activity of $P$. mirabilis urease $^{73}$. In the proposed mode of action, Pta punctures the host cell membrane, causing leakage of the cytosol, osmotic stress and depolymerization of actin filaments; the structural integrity of the cell is therefore compromised, resulting in bladder and kidney damage ${ }^{53,73}$ (FIG. 4). Pta also induces bacterial cell-cell interaction via autoaggregation $^{53,73}$.

$P$. aeruginosa produces elastases, exoenzyme $\mathrm{S}$ A cytoskeletal actin projection at the surface of a cell. In some cases, these actin-powered protrusions are a key factor driving cell motility.
RAC1 function, interfering with lamellopodium formation and inducing membrane ruffle formation. The ADP-ribosyltransfease activity of ExoS targets RHO family proteins (RAS proteins and RalA), affecting cell adherence and morphology ${ }^{76}$. Elastase induces tissue destruction through its protease activity, releasing nutrients (including iron) for continued bacterial growth $^{77}$. Phospholipase $\mathrm{C}$ is an a-toxin that hydrolyses phosphatidylcholine from the host cell membrane, compromising cell integrity and resulting in organ damage $\mathrm{e}^{78-80}$. The expression of all of these virulence factors is regulated by the quorum sensing system ${ }^{81}$. Quorum sensing is activated at high cell density by the accumulation of small molecules called autoinducers. When a threshold level of autoinducers is reached, they bind to transcriptional activator proteins and activate the expression of virulence factors ${ }^{81,82}$ (BOX 1).

Urease. Urease is encoded by several uropathogens, including P. mirabilis ${ }^{53,83}$, S. saprophyticus ${ }^{84}, K$. pneumoniae $^{85}$ and $P$. aureginosa ${ }^{86}$, and is important for colonization and persistence during $P$. mirabilis and S. saprophyticus UTIS $^{83,84}$ (FIG. 4; TABLE 1). This enzyme catalyses the hydrolysis of urea to carbon dioxide and ammonia ${ }^{87}$, resulting in elevated urine $\mathrm{pH}$ and the production of calcium crystals (apatite) and magnesium ammonium phosphate ammoprecipitates (struvite) in urine and on catheters ${ }^{53}$ (FIG. 4). Importantly, the accumulation of ammonia becomes toxic for the uroepithelial cells, inducing direct tissue damage ${ }^{88}$. The $P$. mirabilis urease, one of the best studied ureases involved in UTIs, is a $\mathrm{Ni}^{2+}$-dependent metalloenzyme that is essential for colonization of the bladder and kidneys and promotes the formation of stones ${ }^{23,53,87}$. The $P$. mirabilis urease is induced by urea and is constitutively expressed during growth in urine ${ }^{89}$. This urease is highly active, hydrolysing urea several times faster than those produced by other species, such as Providencia stuartii, Providencia rettgeri, Proteus vulgaris and Morganella morganii $i^{90}$. The high activity level of the $P$. mirabilis enzyme induces rapid crystal formation, and these crystals become trapped within the polysaccharides produced by attached bacterial cells, forming crystalline biofilms on catheters ${ }^{23,89,91}$. The crystalline biofilms provide $P$. mirabilis with protection from the host immune system and antibiotics ${ }^{88}$ (BOX 1; FIC. 4). These structures also block urine drainage from the ureters, potentially resulting in reflux and promoting progression to pyelonephritis, septicaemia and $\operatorname{shock}^{53}$.

Iron scavenging. The bladder environment is limited in iron. Thus, to be able to grow in human urine, uropathogens utilize siderophore systems for iron $\left(\mathrm{Fe}^{3+}\right)$ scavenging; these systems are composed of the siderophore assembly machinery, a siderophore responsible for binding iron and a membrane receptor that internalizes the iron bound to the siderophore ${ }^{92}$ (TABLE 1).

UPEC produces several siderophores ${ }^{93}$, of which two - aerobactin and yersiniabactin - are essential in the urinary $\operatorname{tract}^{93}$ (FIG. 3). Aerobactin is highly expressed, stable at low $\mathrm{pH}$ and displays higher levels of iron binding 
than enterobactin ${ }^{94,95}$. Yersiniabactin is important in biofilm formation in urine and has a protective role against intracellular killing by copper stress, as it sequesters host-derived copper ${ }^{96}$.

Numerous iron-scavenging siderophore systems are utilized by other uropathogens: K. pneumoniae produces enterobactin and aerobactin ${ }^{85} ; P$. mirabilis uses proteobactin and yersiniabactin-related ${ }^{97}$; and $P$. aeruginosa produces pyochelin and pyoverdin ${ }^{86}$ (TABLE 1). Siderophore systems are important potential targets for vaccine development ${ }^{98}$ and for designing small molecules that interfere with their function.

\section{Treatment of urinary tract infections}

UTIs result in considerable economic and public health burdens and substantially affect the life quality of afflicted individuals ${ }^{17}$. Currently, antibiotics — such as trimethoprim sulfamethoxazole, ciprofloxacin and ampicillin - are the most commonly recommended therapeutics for UTIs ${ }^{4}$. However, increasing rates of antibiotic resistance and high recurrence rates threaten to greatly enhance the burden that these common infections place on society. Ideally, alternative therapies will be established that will be recalcitrant to the development of resistance. Many promising approaches are being developed, from leveraging what we have learned about the basic biology of UTI pathogenesis to specifically target virulence pathways. These antivirulence therapeutics should theoretically allow us to effectively neutralize, or 'disarm', the capacity of UTI pathogens to cause disease, without altering the gut commensal microbiota, because antivirulence therapeutics target processes that are critical for UTI pathogenesis but that are not required for the essential processes of growth and cell division (which are the targets of conventional antibiotics).

Below, we discuss the current challenges that have arisen from the emergence of multidrug-resistant bacterial strains and highlight the progress that is being made towards the development of antivirulence therapeutics for UTIs. We also discuss how an understanding of the evolution of bacterial resistance mechanisms and their spread is providing new approaches for the modification and improvement of current therapeutic options.

Multidrug resistance. UTIs are becoming increasingly difficult to treat owing to the widespread emergence of an array of antibiotic resistance mechanisms $\mathrm{s}^{3,4,15,99-102}$ (see Supplementary information S1 (table)). Of particular concern are members of the family Enterobacteriaceae, including E. coli and K. pneumoniae, which have both acquired plasmids encoding extended-spectrum $\beta$-lactamases (ESBLs). These plasmids rapidly spread resistance to third-generation cephalosporins as well as other antibiotics ${ }^{15,99-103}$ (BOX 2). Other Enterobacteriaceae family members produce the class $C \beta$-lactamases (AmpC enzymes) that are active against cephamycin in addition to third-generation cephalosporins, and are also resistant to $\beta$-lactamase inhibitors ${ }^{99-102}$. The expression of AmpC enzymes is also associated with carbapenem resistance in $K$. pneumoniae strains lacking a $42 \mathrm{kDa}$ outer-membrane protein ${ }^{15,99-102}$ (BOX 2).
Multidrug resistance is also common among enterococci, as they are naturally resistant to trimethoprim, clindamycin, cephalosporins and penicillins ${ }^{15,101,102}$. Recently, Enterococcus spp. have developed high-level resistance to glycopeptides, including vancomycin, which is considered to be one of the last lines of defence against multidrug-resistant organisms. Specifically, enterococci evolved resistance to glycopeptides through the expression of vancomycin and teicoplanin A-type resistance (van) genes that encode the penicillin-binding proteins (PBPs) VanA, VanB, VanD, VanE, VanG and VanL ${ }^{101,102}$. The mechanism of resistance for VanA, the most common PBP expressed by enterococci, is to replace the cell wall precursor $\mathrm{D}$-alanine-D-alanine with $\mathrm{D}$-alanine-D-lactose, effectively reducing the binding affinity of vancomycin ${ }^{104}$. The troubling trend towards a high prevalence of multidrug-resistant uropathogens has spurred the development of alternative control measures and treatment options.

Combination therapies. New antimicrobials that are resistant to inactivation by ESBLs are under development for use in combination with new classes of $\beta$-lactamase inhibitors, which target both $\beta$-lactamases and K. pneumoniae carbapenemases (KPCs) ${ }^{105-107}$. These combination therapies have been shown to be effective in vitro against carbapenem-resistant members of the family Enterobacteriaceae. Furthermore, clinical trials involving complicated UTIs revealed that ceftazidime, a third-generation cephalosporin that is active against Gram-positive and Gram-negative organisms, is effective against ESBL- and carbapenemase-producing Gramnegative bacteria when combined with the $\beta$-lactamase inhibitor avibactam ${ }^{105}$. Future studies are needed to test the efficacy of ceftazidime-avibactam against ESBL-, KPC- and AmpC-producing Gram-negative pathogens during infection, as the drug combination has the potential to be effective against a broad range of cephalosporinresistant Enterobacteriaceae family members. Although these antibiotic-inhibitor combinations are promising, the development of resistance to $\beta$-lactamase inhibitors is not well characterized ${ }^{105}$. Moreover, the effectiveness of specific antibiotic-inhibitor therapies is dependent on the antimicrobial-resistance patterns encoded by each pathogen, as the expression of certain combinations of ESBLs and carbapenemases can provide resistance to an antibiotic-inhibitor therapy ${ }^{105-107}$. For example, the combination of BAL30072-BAL29880-clavulanate (two $\beta$-lactam antibiotics and a $\beta$-lactamase inhibitor) is effective against many carbapenem-resistant Enterobacteriaceae family members, but K. pneumoniae strains that typically produce KPCs and SHVs (another type of ESBL), or AmpC enzymes are resistant ${ }^{106}$. Therefore, it is crucial to know which antibiotic mechanisms are available to a specific uropathogen in order to determine an effective treatment.

Vaccines targeting bacterial adhesion. As adherence has a key role at nearly every step of UTI pathogenesis, one attractive strategy for the development of antivirulence therapies, including vaccines, has been to target CUP pili. As a general rule, vaccination with whole pili has been ineffective at generating an antibody response that can 


\section{Box 2 | Antibiotic resistance}

Multidrug-resistant uropathogenic organisms are becoming an expanding public health threat, as Enterobacteriaceae family members increasingly acquire extended-spectrum $\beta$-lactamases (ESBLs) such as cefotaximases (CTX-Ms) and oxacillinases (OXAs), AmpC-type $\beta$-lactamases and carbapenemases.

ESBLs

Originating in Klebsiella pneumoniae and Escherichia coli, ESBLs are now prevalent throughout the Enterobacteriaceae family, as frequent use of cephalosporins in the nosocomial setting and the carriage of ESBL-encoding genes on transferrable elements together create an ideal environment for the selection of antibiotic resistance ${ }^{99,102}$. ESBLs are plasmid-encoded or chromosomally encoded $\beta$-lactamases with broad activity against penicillins and cephalosporins. They function by splitting the amide bond of the $\beta$-lactam ring, thus inactivating $\beta$-lactam antibiotics ${ }^{102}$. Troublingly, ESBLs are encoded on plasmids that typically carry other resistance genes which provide activity against aminoglycosides, sulfonamides and quinolones, making the bacteria that acquire these plasmids multidrug resistant ${ }^{101,102}$.

\section{CTX-Ms}

The plasmids encoding the ESBLs CTX-Ms form a new plasmid phylum that is phylogenetically distinct from other plasmid-encoded $\beta$-lactamases. CTX-Ms are active against narrow-, broad- and extended-spectrum penicillins, classical and extended-spectrum cephalosporins, and monobactams $\mathrm{s}^{99,102,103}$. Notably, they also confer high-level cefotaxime resistance ${ }^{99,103}$. CTX-Ms are the most prevalent $\beta$-lactamases in community-associated isolates and are typically encoded on plasmids with other resistance genes ${ }^{102}$. CTX-Ms efficiently hydrolyse the $\beta$-lactam ring via nucleophilic attack of a ring carbonyl carbon by a conserved serine in the $\beta$-lactamase, resulting in a ring-opened product that is inactive ${ }^{140}$.

\section{OXAs}

OXAs are ESBLs that are typically encoded by plasmids and mediate resistance to ampicillin, cephalothin, oxacillin and cloxacillin by hydrolysing the $\beta$-lactam rings ${ }^{99,103}$. In addition, OXAs are characterized by their ability to resist the $\beta$-lactamase inhibitor clavulante ${ }^{103}$. To date, OXAs have been shown to be expressed only in Pseudomonas aeruginosa $a^{99,103}$.

\section{AmpC enzymes}

The chromosomally encoded AmpC enzymes hydrolyse penicillins, third-generation and extended-spectrum cephalosporins, and cephamycins, and are resistant to $\beta$-lactamase inhibitors, including clavulanate ${ }^{99,102}$. AmpC expression is induced in response to $\beta$-lactams, cephamycin and cephalosporin exposure.

\section{Carbapenemases}

Carbapenemases are ESBLs that confer the ability to inactive carbapenems in addition to penicillins and extended-spectrum cephalosporins $s^{99,101,102}$. The two most clinically relevant carbapenemases, K. pneumoniae (serine) carbapenemase (KPC) and New Delhi metallo- $\beta$-lactamase (NDM-1), originated in K. pneumoniae and rapidly spread throughout the Enterobacteriaceae family, creating carbapenem-resistant Enterobacteriaceae $(\mathrm{CRE})^{15,99,101,102}$. The broad activity of carbapenemases confers resistance against a wide range of extended-spectrum $\beta$-lactam antibiotics, particularly carbapenem.

protect against UTIs. However, adhesin-based vaccines have been shown to be effective at blocking host-pathogen interactions, thus preventing the establishment of disease $\mathrm{e}^{108-112}$. Experiments using mouse and cynomolgus monkey models of UTIs determined that immunization with PapD-PapG or FimC-FimH chaperone-adhesin complexes protected against UTIs ${ }^{108-112}$. The effectiveness of the FimC-FimH vaccine was shown to be due, in large part, to antibodies that block the function of FimH in bladder colonization ${ }^{110}$. Furthermore, the anti-FimH antibodies did not seem to alter the E. coli niche in the gut microbiota ${ }^{109}$. Modifications of this vaccine are currently under development, with the aim of inducing greater immune stimulation ${ }^{108,112}$. For example, one approach has been to fuse FimH to the flagellin FliC in order to induce a more substantial acute inflammatory response, which functions through TLR4 signalling via the MYD88 pathway $^{112}$. A Phase I clinical trial began in January 2014 to evaluate the efficacy of a FimC-FimH vaccine using a synthetic analogue of monophosphoryl lipid $\mathrm{A}$ as the adjuvant.

In addition to the UPEC adhesins, adhesins from P. mirabilis and E. faecalis have also been used as vaccine targets ${ }^{60,113}$. In a mouse model of UTI, vaccination with the $P$. mirabilis $\mathrm{MR} / \mathrm{P}$ pilus adhesin, $\mathrm{MrpH}$, reduced bacterial burdens compared with those of unvaccinated controls, similar to the results observed with UPEC in the FimH vaccine trials ${ }^{110,113}$. Moreover, a vaccine strategy that is efficacious against E. faecalis CAUTIs is being developed based on vaccination with the Ebp pilus adhesin, EbpA. This strategy induced high antibody titers and reduced bacterial burdens in a mouse model of CAUTI ${ }^{60}$. In conclusion, adhesin-based vaccines represent a promising area for the development of therapeutics against uropathogens. Thus, understanding the molecular basis of host-pathogen interactions is crucial for vaccine development strategies.

Vaccines targeting bacterial toxins and proteases. The UPEC pore-forming toxin HlyA has also received attention as a potential vaccine target and was evaluated in a mouse model of pyelonephritis to assess protection against renal damage ${ }^{114,115}$. Vaccination with HlyA reduced the incidence of renal scaring compared with controls; however, it did not protect against UPEC colonization of the kidneys ${ }^{115}$. In addition, in a mouse model of UTI, vaccination with the P. mirabilis haemolysin, HpmA, did not provide protection against bacterial colonization $^{116}$. However, vaccination with Pta, an alkaline protease with toxic effects towards epithelial cells, displayed promising results in a mouse model of UTI, protecting against upper UTI, although bacterial burdens in the bladder remained unaffected ${ }^{116}$. Thus, although haemolysins and proteases might provide effective vaccine targets for preventing upper UTIs, additional studies are needed to determine the effectiveness of these enzymes as targets for vaccines.

Vaccines targeting siderophores. Iron acquisition systems have shown great promise as targets for vaccine development because uropathogens require a source of iron during colonization and persistence. Furthermore, siderophore and haem acquisition systems have been shown to be upregulated during experimental infection, as well as in the urine of women with a $\mathrm{UTI}^{86,94,97,98}$. These parameters sparked vaccine development based on ferric yersiniabactin uptake receptor (FyuA), haem acquisition protein (Hma), iron uptake transport aerobactin receptor (IutA) and the siderophore receptor iron-responsive element A (IreA) ${ }^{98}$. Vaccination with FyuA and Hma protected mice against pyelonephritis ${ }^{98,117}$, whereas vaccination with IutA and IreA reduced bladder colonization in mice, confirming the importance of these proteins during infection ${ }^{98,117}$. Interestingly, the differential tissue-specific protection seen with these four proteins suggests that these systems have different roles or expression profiles in different niches, including the bladder or kidneys. 
Vaccinations with other siderophore systems in mouse models of UTI, including the iron receptors FitA and $\mathrm{ChuA}^{98}$, were not protective against infection and were correlated, to a large extent, with lower antigen-specific humoral responses during experimental UTI. These studies suggest that effective siderophore-based vaccines function in part by preventing cognate siderophore uptake, as is the case with FyuA, Hma, IutA and Ire $\mathrm{A}^{98,117}$, making this an exciting area of therapeutic development against UTIs.

Small molecules targeting urease. Several urease inhibitors have been developed as potential drugs for UTI treatment, with varying results ${ }^{89}$. Many of the early inhibitors were active against ureases from several different bacterial species, including Helicobacter pylori, P. mirabilis and S. saprophyticus, and many of these inhibitors showed great promise, as they had low binding and inhibitory concentrations. The best characterized urease inhibitor, acetohydroxamic acid (AHA), even had some success in treating UTIs caused by urease-producing organisms; this inhibitor works by preventing urine alkalization and was approved by the FDA in 1983 (REF. 89). However, many of these inhibitors had severe side effects related to toxicity. For example, AHA resulted in teratogenicity, as well as psychoneurological and musculo-integumentary effects. Subsequent studies showed that derivatives of AHA also had considerable inhibitory properties, but again, these compounds had mutagenic properties that made them undesirable therapeutics ${ }^{89}$. Another group of urease inhibitors, the phosphoramidites, exhibited potent activity against $P$. mirabilis urease and were effective in a mouse model of infection. However, this class of compounds displayed low stability in the low $\mathrm{pH}$ of gastric juice, making them impractical ${ }^{89}$. Finally, the heterocyclic compounds termed benzimidazoles have garnered much attention because they function as proton pump inhibitors that irreversibly inactivate ATPase systems ${ }^{118}$. These compounds are currently the standard treatment for peptic ulcers and gastroesophageal reflux disease ${ }^{89}$. Benzimidazoles interact with the gastric hydrogen potassium ATPase, thereby inactivating them and effectively limiting the disease $\mathrm{e}^{118}$. Interestingly, benzimidazoles also bind to the urease metallocentre, effectively blocking the active site of the enzyme through steric hindrance ${ }^{89}$. Benzimidazoles also have a bactericidal activity against H. pylori, and this is not mediated by urease inhibition, indicating that these compounds have a more general bactericidal effect ${ }^{89,119}$. Great strides have been made to identify and characterize urease inhibitors, but more work is needed to bring these potential treatments to the market.

The capability of a compound to cause fetal malformation.

Musculo-integumentary Refers to the interaction between the muscular and integumentary systems. The muscular system is composed by the skeletal, smooth and cardiac muscles, whereas the skin, hair, nails and other specialized structures form the integumentary system.
Small molecules targeting bacterial adhesion. Our detailed understanding of pilus assembly and pilus-receptor binding has opened the door to the development of two classes of small, rationally designed synthetic compounds to inhibit pili: mannosides, which inhibit pilus function; and pilicides, which inhibit pilus assembly. Targeting CUP pilus function or assembly has therapeutic potential, as it should block UPEC colonization, invasion and biofilm formation, thus preventing disease $\mathrm{e}^{30,31,120,121}$.
Pilicides were originally developed to specifically inhibit the assembly of UPEC type 1 pili. They have a 2-pyridone scaffold ${ }^{28,30,31,120}$ and function by selectively targeting and interfering with crucial chaperone-usher interactions. Further studies have been carried out to investigate their broad spectrum of activity against other CUP pili ${ }^{122}$. A recent analysis of 35 Escherichia spp. genomes and 132 plasmids identified a total of 458 CUP operons, representing 38 distinct CUP pilus types on the basis of usher phylogeny ${ }^{25}$. A single Escherichia sp. genome can have as many as 16 distinct, intact CUP operons $^{25}$, suggesting that compounds which target CUP pili by disrupting their assembly would potentially exhibit broad-spectrum activity. For example, pilicide ec240 was found to disrupt several virulence-associated pili, including type 1 pili, $\mathrm{P}$ pili and S pili, as well as flagellar motility ${ }^{122}$. The effect of ec 240 on the transcriptome and proteome of the cystitis isolate E. coli UTI89 revealed that the most downregulated genes after growth in the presence of ec 240 were the type 1 pilus genes. Type 1 pilus expression is controlled by inversion of the type 1 fimbriae promoter element (fimS), which can oscillate between phase ON and phase OFF orientations. ec240 induced the fimS phase OFF orientation and increased the expression of the transcriptional regulators S-fimbrial switch regulatory protein $(\mathrm{SfaB})$ and $\mathrm{P}$ pilus regulatory protein PapB, which have been shown to promote a fimS phase OFF orientation ${ }^{122}$. Thus, the potency of pilicide ec240 is largely due to its ability to induce a phase OFF orientation of the type 1 pilus promoter, rather than any interference with chaperone-usher interactions. Additional work revealed that other pilicides also inhibit the production of Dr pili, another type of UPEC CUP pili that are known to be important in pyelonephritis in mice and humans ${ }^{30,33}$. Furthermore, pilicides have been shown to disrupt CUP pilus biogenesis in K. pneumoniae and also in Haemophilus influenzae (a finding that has important implications for otitis media $)^{24,29}$. Thus, pilicides represent an exciting class of antivirulence molecules with the potential to target a broad spectrum of pathogens that utilize CUP pili in attachment and the establishment of infection. Future studies using mouse models of UTIs and CAUTIs to investigate the role of CUP pili in Gram-negative bacterial infections, as well as the efficacy and bioavailability of pilicides as therapeutics, will unravel the potential of this class of molecules.

Mannosides, which are FimH receptor analogues, have been developed to bind FimH with high affinity and block FimH binding to mannosylated receptors $^{35,121,123-125}$. Mannosides are potent FimH antagonists that offer a promising therapeutic opportunity for the treatment and prevention of UTIs by interrupting key host-pathogen interactions ${ }^{123-125}$. Studies in mouse models have demonstrated the potential of mannosides as novel therapeutic strategies against UTIs: mannosides are orally bioavailable; they are potent and fast-acting therapeutics in treating and preventing UTIs; they function by preventing bladder colonization and invasion; they are effective against multidrug-resistant UPEC; they potentiate antibiotic efficacy; and they are effective against established UTIs and CAUTIs ${ }^{35,121,124,125}$. 
Interestingly, the adhesin FimH undergoes a substantial structural change during transit across the usher pore, such that the receptor-binding lectin domain bends approximately $37^{\circ}$ with respect to the pilin domain. Thus, FimH adopts an elongated conformation before transport across the usher pore ${ }^{32,126}$, whereas the lectin domain swings closer to the pilin domain after transport $^{32}$. The two forms of the lectin domain have important implications for binding and pathogenesis: the elongated conformation binds mannose with a significantly higher affinity than the compact form ${ }^{126}$. Residues that control these conformational transitions have been shown to be under positive selection, and pathoadaptive alleles of FimH have subsequently been identified ${ }^{126-128}$. Thus, we now understand how protein-protein interactions and ligand binding can regulate a dynamic conformational equilibrium in the receptor-binding domain of FimH, and this is revealing unexpected insights into UTI pathogenesis and potentiating mannoside development. This unravelling of the dynamics of how allostery governs CUP pilus assembly and function is providing valuable information about macromolecular protein assembly and virulence in Gram-negative pathogens and is spawning new ways of thinking about drug development.

\section{Outlook}

UTIs are some of the most common bacterial infections, resulting in billions of dollars in health care costs annually ${ }^{1}$. Both the numerous uropathogens, which encode a wide range of virulence factors, and the spread of antimicrobial resistance threaten the only effective treatment option available - antibiotics ${ }^{15,17}$. Moreover, high rates of recurrent UTIs suggest that antibiotics are not an effective therapy for all UTIs. Intensive studies have laid the foundations for conducting translational research that can identify essential mechanisms of virulence and provide evidence to guide the development of UTI treatments and prophylactics that are optimized against uropathogens and that do not alter the normal microflora. The identification of virulence determinants - specifically, those that are essential for initial attachment, including adhesins, and for the subsequent establishment of disease, including siderophores and urease - has allowed the development of targeted therapies that effectively neutralize pathogenic bacteria and prevent disease in animal models. By targeting the initial steps of infection - either through chemical compounds, such as mannosides and pilicides, or by vaccination with adhesins or siderophore receptors - these therapies aim to prevent uropathogens from gaining a foothold in the urinary tract.

Although great strides have been made in developing new strategies that might one day be of value in the treatment and prevention of UTIs, more work is needed. Although the FimH vaccine is in Phase I clinical trials, many of the other potential therapies, including mannosides, pilicides, and vaccines against siderophores, toxins and pili, are still in the preclinical stages of development and have been tested only in animal models. Importantly, the impact of these strategies on the endogenous microbiota should be considered. For example, although these antivirulence therapeutics are not expected to greatly affect the microbiota (as Enterobacteriaceae family members make up only a small portion of the gut flora), only the FimH vaccine has so far been demonstrated to have no effect on the normal composition of the gut microbiota ${ }^{109}$.

Finally, substantial effort should be put into setting up future clinical trials, which will be essential for translating these novel antivirulence therapies into new treatments that reduce the suffering associated with UTIs.
1. Stamm, W. E. \& Norrby, S. R. Urinary tract infections: disease panorama and challenges. J. Infect. Dis. 183 (Suppl. 1), S1-S4 (2001).

2. Schappert, S. M. \& Rechtsteiner, E. A. Ambulatory medical care utilization estimates for 2007. Vital Health Stat. 13, 1-38 (2011).

3. Foxman, B. Urinary tract infection syndromes: occurrence, recurrence, bacteriology, risk factors, and disease burden. Infect. Dis. Clin. North Am. 28, 1-13 (2014).

This paper presents the most recent information about UTIs and their socioeconomic impact.

4. Foxman, B. The epidemiology of urinary tract infection. Nature Rev. Urol. 7, 653-660 (2010).

Hooton, T. M. Uncomplicated urinary tract infection. New Engl. J. Med. 366, 1028-1037 (2012).

6. Nielubowicz, G. R. \& Mobley, H. L. Host-pathogen interactions in urinary tract infection. Nature Rev. Urol. 7, 430-441 (2010).

This review compares the strategies used by two important uropathogens, $E$. coli and $P$. mirabilis, the host response to each pathogen, and the current treatments and therapies to prevent UTIs.

7. Hannan, T. J. et al. Host-pathogen checkpoints and population bottlenecks in persistent and intracellular uropathogenic Escherichia coli bladder infection. FEMS Microbiol. Rev. 36, 616-648 (2012).

8. Lichtenberger, P. \& Hooton, T. M. Complicated urinary tract infections. Curr. Infect. Dis. Rep. 10, 499-504 (2008).

9. Levison, M. E. \& Kaye, D. Treatment of complicated urinary tract infections with an emphasis on drugresistant Gram-negative uropathogens. Curr. Infect. Dis. Rep. 15, 109-115 (2013).
10. Lo, E. et al. Strategies to prevent catheter-associated urinary tract infections in acute care hospitals: 2014 update. Infect. Control Hosp. Epidemiol. 35 464-479 (2014)

11. Chenoweth, C. E., Gould, C. V. \& Saint, S. Diagnosis, management, and prevention of catheter-associated urinary tract infections. Infect. Dis. Clin. North Am. 28 , 105-119 (2014).

12. Kline, K. A., Schwartz, D. J., Lewis, W. G. Hultgren, S. J. \& Lewis, A. L. Immune activation and suppression by group B Streptococcus in a murine model of urinary tract infection. Infect. Immun. 79, 3588-3595 (2011).

13. Ronald, A. The etiology of urinary tract infection: traditional and emerging pathogens. Am. J. Med. 113 (Suppl. 1A), 14S-19S (2002).

14. Fisher, J. F., Kavanagh, K., Sobel, J. D., Kauffman, C. A. \& Newman, C. A. Candida urinary tract infection: pathogenesis. Clin. Infect. Dis. 52 (Suppl. 6), S437-S451 (2011).

15. Chen, Y. H., Ko, W. C. \& Hsueh, P. R. Emerging resistance problems and future perspectives in pharmacotherapy for complicated urinary tract infections. Expert Opin. Pharmacother. 14, 587-596 (2013).

This paper highlights the emerging resistance among bacterial pathogens, the problems we face in combating these resistant bacteria and potential effective agents for the treatment of UTIs caused by multidrug-resistant pathogens.

16. Jacobsen, S. M., Stickler, D. J., Mobley, H. L. \& Shirtliff, M. E. Complicated catheter-associated urinary tract infections due to Escherichia coli and Proteus mirabilis. Clin. Microbiol. Rev. 21, 26-59 (2008).
17. Kostakioti, M., Hultgren, S. J. \& Hadjifrangiskou, M Molecular blueprint of uropathogenic Escherichia coli virulence provides clues toward the development of anti-virulence therapeutics. Virulence 3, 592-594 (2012).

18. Subashchandranose, S. et al. Host-specific induction of Escherichia coli fitness genes during human urinary tract infection. Proc. Natl Acad. Sci. USA 111,18327-18332 (2014).

19. Khandelwal, P., Abraham, S. N. \& Apodaca, G. Cell biology and physiology of the uroepithelium. Am. J. Physiol. Renal Physiol. 297, F1477-F1501 (2009).

20. Lee, G. Uroplakins in the lower urinary tract. Int. Neurourol. J. 15, 4-12 (2011).

21. Eto, D. S., Jones, T. A., Sundsbak, J. L. \& Mulvey, M. A. Integrin-mediated host cell invasion by type 1-piliated uropathogenic Escherichia coli. PLoS Pathog. 3, e100 (2007).

22. Niveditha, S., Pramodhini, S., Umadevi, S., Kumar, S. $\&$ Stephen, $\mathrm{S}$. The isolation and the biofilm formation of uropathogens in the patients with catheter associated urinary tract infections (UTIs). J. Clin. Diagn. Res. 6, 1478-1482 (2012).

23. Jacobsen, S. M. $\propto$ Shirtliff, M. E. Proteus mirabilis biofilms and catheter-associated urinary tract infections. Virulence 2, 460-465 (2011). This paper briefly outlines the steps of $P$. mirabilis crystalline-biofilm formation during CAUTIs.

24. Kline, K. A., Dodson, K. W., Caparon, M. G. \& Hultgren, S. J. A tale of two pili: assembly and function of pili in bacteria. Trends Microbiol. 18, 224-232 (2010).

25. Wurpel, D. J., Beatson, S. A., Totsika, M., Petty, N. K. $\&$ Schembri, M. A. Chaperone-usher fimbriae of Escherichia coli. PLOS ONE 8, e52835 (2013). 
26. Waksman, G. \& Hultgren, S. J. Structural biology of the chaperone-usher pathway of pilus biogenesis. Nature Rev. Microbiol. 7, 765-774 (2009). This review presents the most current, in-depth understanding of how pili are assembled through the chaperone-usher pathway.

27. Vallet, I., Olson, J. W., Lory, S., Lazdunski, A. \& Filloux, A. The chaperone/usher pathways of Pseudomonas aeruginosa: identification of fimbrial gene clusters (cup) and their involvement in biofilm formation. Proc. Natl Acad. Sci. USA 98, 6911-6916 (2001).

28. Chorell, E. et al. Mapping pilicide anti-virulence effect in Escherichia coli, a comprehensive structureactivity study. Bioorg. Med. Chem. 20, 31 28-3142 (2012).

29. Thanassi, D. G., Saulino, E. T. \& Hultgren, S. J. The chaperone/usher pathway: a major terminal branch of the general secretory pathway. Curr. Opin. Microbiol. 1, 223-231 (1998)

30. Piatek, R. et al. Pilicides inhibit the FGL chaperone/ usher assisted biogenesis of the Dr fimbrial polyadhesin from uropathogenic Escherichia coli. BMC Microbiol. 13, 131 (2013).

This paper provides a brief overview of two similar CUP pilus assembly pathways and shows that antivirulence compounds (pilicides) which were originally designed to specifically target one pathway have broad-spectrum activity against both CUP pilus pathways in $E$. coli.

31. Dang, H. T. et al. Syntheses and biological evaluation of 2-amino-3-acyl-tetrahydrobenzothiophene derivatives; antibacterial agents with antivirulence activity. Org. Biomol. Chem. 12, 1942-1956 (2014).

32. Geibel, S., Procko, E., Hultgren, S. J., Baker, D. \& Waksman, G. Structural and energetic basis of folded protein transport by the FimD usher. Nature 496 243-246 (2013).

33. Wright, K J \& Hultoren, S J Sticky fibers and uropathogenesis: bacterial adhesins in the urinary tract. Future Microbiol. 1, 75-87 (2006).

34. Hadjifrangiskou, M. et al. Transposon mutagenesis identifies uropathogenic Escherichia coli biofilm factors. J. Bacteriol. 194, 6195-6205 (2012).

35. Guiton, P. S. et al. Combinatorial small-molecule therapy prevents uropathogenic Escherichia coli catheter-associated urinary tract infections in mice. Antimicrob. Agents Chemother. 56, 4738-4745 (2012).

36. Martinez, J. J. \& Hultgren, S. J. Requirement of Rhofamily GTPases in the invasion of type 1-piliated uropathogenic Escherichia coli. Cell. Microbiol. 4 19-28 (2002)

37. Song, J. et al. TLR4-mediated expulsion of bacteria from infected bladder epithelial cells. Proc. Natl Acad. Sci. USA 106, 14966-14971 (2009).

38. Anderson, G. G. et al. Intracellular bacterial biofilm like pods in urinary tract infections. Science 301 , 105-107 (2003)

This is the first paper to describe the intracellular cycle of a uropathogen and its importance for persistance.

39. Hannan, T. J., Mysorekar, I. U., Hung, C. S., IsaacsonSchmid, M. L. \& Hultgren, S. J. Early severe inflammatory responses to uropathogenic $E$. coli predispose to chronic and recurrent urinary tract infection. PLoS Pathog. 6, e1001042 (2010)

40. Kostakioti, M., Hadjifrangiskou, M. \& Hultgren, S. J. Bacterial biofilms: development, dispersal, and therapeutic strategies in the dawn of the postantibiotic era. Cold Spring Harb. Perspect. Med. 3, a010306 (2013).

This review details the importance of biofilm formation for the survival and persistance of different pathogens and the threat that represents in clinical settings. In addition, it discusses novel alternative strategies for the prevention of biofilm formation

41. Rosen, D. A., Hooton, T. M., Stamm, W. E., Humphrey, P. A. \& Hultgren, S. J. Detection of intracellular bacterial communities in human urinary tract infection. PLoS Med. 4, e329 (2007).

42. Robino, L. et al. Intracellular bacteria in the pathogenesis of Escherichia coli urinary tract infection in children. Clin. Infect. Dis. 59, e 158-e 164 (2014).

43. Schwartz, D. J., Chen, S. L., Hultgren, S. J. \& Seed, P. C. Population dynamics and niche distribution of uropathogenic Escherichia coli during acute and chronic urinary tract infection. Infect. Immun. 79 , 4250-4259 (2011).
44. Blango, M. G., Ott, E. M., Erman, A., Veranic, P. \& Mulvey, M. A. Forced resurgence and targeting of intracellular uropathogenic Escherichia coli reservoirs. PLOS ONE 9, e93327 (2014)

45. Rice, J. C. et al. Pyelonephritic Escherichia coli expressing $\mathrm{P}$ fimbriae decrease immune response of the mouse kidney. J. Am. Soc. Nephrol. 16 3583-3591 (2005)

46. Ashkar, A. A., Mossman, K. L., Coombes, B. K. Gyles, C. L. \& Mackenzie, R. FimH adhesin of type 1 fimbriae is a potent inducer of innate antimicrobial responses which requires TLR4 and type 1 interferon signalling. PLoS Pathog. 4, e1000233 (2008)

47. Gerlach, G. F., Clegg, S. \& Allen, B. L. Identification and characterization of the genes encoding the type-3 and type- 1 fimbrial adhesins of Klebsiella pneumoniae. J. Bacteriol. 171, 1262-1270 (1989).

48. Stahlhut, S. G. et al. Comparative structure-function analysis of mannose-specific FimH adhesins from Klebsiella pneumoniae and Escherichia coli. J. Bacteriol 191, 6592-6601 (2009).

49. Rosen, D. A. et al. Molecular variations in Klebsiella pneumoniae and Escherichia coli FimH affect function and pathogenesis in the urinary tract. Infect. Immun. 76, 3346-3356 (2008)

50. Rosen, D. A. et al. Utilization of an intracellular bacterial community pathway in Klebsiella pneumoniae urinary tract infection and the effects of FimK on type 1 pilus expression. Infect. Immun. 76 , 3337-3345 (2008)

51. Murphy, C. N., Mortensen, M. S., Krogfelt, K. A. \& Clegg, S. Role of Klebsiella pneumoniae type 1 and type 3 fimbriae in colonizing silicone tubes implanted into the bladders of mice as a model of catheterassociated urinary tract infections. Infect. Immun. $\mathbf{8 1}$ 3009-3017 (2013)

52. Struve, C., Bojer, M. \& Krogfelt, K. A. Characterization of Klebsiella pneumoniae type 1 fimbriae by detection of phase variation during colonization and infection and impact on virulence. Infect. Immun. 76 4055-4065 (2008)

53. Armbruster, C. E. \& Mobley, H. L. Merging mythology and morphology: the multifaceted lifestyle of Proteus mirabilis. Nature Rev. Microbiol. 10, 743-754 (2012).

54. Arias, C. A. \& Murray, B. E. The rise of the Enterococcus: beyond vancomycin resistance. Nature Rev. Microbiol. 10, 266-278 (2012) This is a comprehensive review of the epidemiology, pathogenesis and mechanism of antimicrobial resistance of Enterococcus spp. This review also outlines how Enterococcus spp. are becoming a challenging nosocomial problem.

55. Guiton, P. S., Hung, C. S., Hancock, L. E., Caparon, M. G. \& Hultgren, S. J. Enterococcal biofilm formation and virulence in an optimized murine model of foreign body-associated urinary tract infections. Infect. Immun. 78, 4166-4175 (2010).

56. Nielsen, H. V. et al. The metal ion-dependent adhesion site motif of the Enterococcus faecalis EbpA pilin mediates pilus function in catheter-associated urinary tract infection. mBio 3, e00177-12 (2012).

57. Goble, N. M., Clarke, T. \& Hammonds, J. C. Histological changes in the urinary bladder secondary to urethral catheterisation. $\mathrm{Br}$. J. Urol. 63, 354-357 (1989).

58. Glahn, B. E. Influence of drainage conditions on mucosal bladder damage by indwelling catheters. I. Pressure study. Scand. J. Urol. Nephrol. 22, 87-92 (1988)

59. Guiton, P. S., Hannan, T. J., Ford, B. Caparon, M. G. \& Hultgren, S. J. Enterococcus faecalis overcomes foreign body-mediated inflammation to establis urinary tract infections. Infect. Immun. 81, 329-339 (2013)

60. Flores-Mireles, A. L., Pinkner, J. S., Caparon, M. G. \& Hultgren, S. J. EbpA vaccine antibodies block binding of Enterococcus faecalis to fibrinogen to prevent catheter-associated bladder infection in mice. Sci. Transl. Med. 6, 254 ra1 27 (2014)

This is the first study to dissect the mechanism of $E$. faecalis infection during a CAUTI; this work led to the development of a vaccine that prevents infection in a mouse model of a CAUTI.

61. Nielsen, H. V. et al. Pilin and sortase residues critical for endocarditis- and biofilm-associated pilus biogenesis in Enterococcus faecalis. J. Bacteriol. 195, 4484-4495 (2013)

62. Dhakal, B. K. \& Mulvey, M. A. The UPEC pore-forming toxin $\alpha$-hemolysin triggers proteolysis of host proteins to disrupt cell adhesion, inflammatory, and survival pathways. Cell Host Microbe 11, 58-69 (2012).
63. Nagamatsu, K. et al. Dysregulation of Escherichia coli $\alpha$-hemolysin expression alters the course of acute and persistent urinary tract infection. Proc. Natl Acad. Sci. USA 112, E871-E880 (2015).

64. Mulvey, M. A. et al. Induction and evasion of host defenses by type 1-piliated uropathogenic Escherichia coli. Science 282, 1494-1497 (1998).

65. Justice, S. S. \& Hunstad, D. A. UPEC hemolysin: more than just for making holes. Cell Host Microbe 11, 4-5 (2012)

66. Hannan, T. J et al. LeuX tRNA-dependent and -independent mechanisms of Escherichia coli pathogenesis in acute cystitis. Mol. Microbiol. 67 116-128 (2008)

67. Garcia, T. A., Ventura, C. L., Smith, M. A., Merrell, D. S. \& O'Brien, A. D. Cytotoxic necrotizing factor 1 and hemolysin from uropathogenic Escherichia coli elicit different host responses in the murine bladder. Infect. Immun. 81, 99-109 (2013)

68. Landraud, L. et al. E. coli CNF1 toxin: a two-in-one system for host-cell invasion. Int. J. Med. Microbiol. 293, 513-518 (2004).

69. Piteau, M. et al. Lu/BCAM adhesion glycoprotein is a receptor for Escherichia coli cytotoxic necrotizing factor 1 (CNF1). PLoS Pathog. 10, e1003884 (2014).

70. Doye, A. et al. CNF1 exploits the ubiquitin-proteasome machinery to restrict Rho GTPase activation for bacterial host cell invasion. Cell 111, 553-564 (2002).

71. Miraglia, A. G. et al. Cytotoxic necrotizing factor prevents apoptosis via the Akt/lkB kinase pathway: role of nuclear factor-kB and $\mathrm{Bcl}-2$. Mol. Biol. Cell 18 2735-2744 (2007)

72. Cestari, S. E. et al. Molecular detection of HpmA and HlyA hemolysin of uropathogenic Proteus mirabilis. Curr. Microbiol. 67, 703-707 (2013)

73. Alamuri, P. \& Mobley, H. L. A novel autotransporter of uropathogenic Proteus mirabilis is both a cytotoxin and an agglutinin. Mol. Microbiol. 68, 997-1017 (2008).

74. Mittal, R., Khandwaha, R. K., Gupta, V., Mittal, P. K. \& Harjai, K. Phenotypic characters of urinary isolates of Pseudomonas aeruginosa and their association with mouse renal colonization. Indian J. Med. Res. 123 , 67-72 (2006)

75. Mittal, R., Sharma, S., Chhibber, S. \& Harjai, K. Iron dictates the virulence of Pseudomonas aeruginosa in urinary tract infections. J. Biomed. Sci. 15, 731-741 (2008).

76. Rocha, C. L., Coburn, J., Rucks, E. A. \& Olson, J. C. Characterization of Pseudomonas aeruginosa exoenzyme $\mathrm{S}$ as a bifunctional enzyme in J774A.1 macrophages. Infect. Immun. 71, 5296-5305 (2003).

77. Cathcart, G. R. et al. Novel inhibitors of the Pseudomonas aeruginosa virulence factor LasB: a potential therapeutic approach for the attenuation of virulence mechanisms in pseudomonal infection. Antimicrob. Agents Chemother. 55, 2670-2678 (2011)

78. Meyers, D. J. et al. In vivo and in vitro toxicity of phospholipase C from Pseudomonas aeruginosa. Toxicon 30, 161-169 (1992).

79. Wargo, M. J. et al. Hemolytic phospholipase C inhibition protects lung function during Pseudomonas aeruginosa infection. Am. J. Respir. Crit. Care Med. 184, 345-354 (2011).

80. Berka, R. M. \& Vasil, M. L. Phospholipase C (heatlabile hemolysin) of Pseudomonas aeruginosa purification and preliminary characterization J. Bacteriol. 152, 239-245 (1982)

81. Senturk, S., Ulusoy, S., Bosgelmez-Tinaz, G. \& Yagci, A. Quorum sensing and virulence of Pseudomonas aeruginosa during urinary tract infections. J. Infect. Dev. Ctries 6, 501-507 (2012)

82. Mittal, R., Aggarwal, S., Sharma, S., Chhibber, S. \& Harjai, K. Urinary tract infections caused by Pseudomonas aeruginosa: a mini review. J. Infect. Publ. Health 2, 101-111 (2009).

83. Li, X. et al. Visualization of Proteus mirabilis within the matrix of urease-induced bladder stones during experimental urinary tract infection. Infect. Immun. 70, 389-394 (2002)

84. Gatermann, S., John, J. \& Marre, R. Staphylococcus saprophyticus urease: characterization and contribution to uropathogenicity in unobstructed urinary tract infection of rats. Infect. Immun. $\mathbf{5 7}$ 110-116 (1989). 
85. Podschun, R. \& Ullmann, U. Klebsiella spp. as nosocomial pathogens: epidemiology, taxonomy, typing methods, and pathogenicity factors. Clin. Microbiol. Rev. 11, 589-603 (1998)

86. Visca, P. et al. Virulence determinants in Pseudomonas aeruginosa strains from urinary tract infections. Epidemiol. Infect. 108, 323-336 (1992).

87. Griffith, D. P., Musher, D. M. \& Itin, C. Urease. The primary cause of infection-induced urinary stones. Invest. Urol. 13, 346-350 (1976).

88. Coker, C., Poore, C. A., Li, X. \& Mobley, H. L. Pathogenesis of Proteus mirabilis urinary tract infection. Microbes Infect. 2, 1497-1505 (2000).

89. Kosikowska, P. \& Berlicki, L. Urease inhibitors as potential drugs for gastric and urinary tract infections: a patent review. Expert Opin. Ther. Pat. 21, 945-957 (2011).

90. Jones, B. D. \& Mobley, H. L. Genetic and biochemical diversity of ureases of Proteus, Providencia, and Morganella species isolated from urinary tract infection. Infect. Immun. 55, 2198-2203 (1987).

91. Stickler, D. J. Clinical complications of urinary catheters caused by crystalline biofilms: something needs to be done. J. Intern. Med. 276, 120-129 (2014).

92. Caza, M. \& Kronstad, J. W. Shared and distinct mechanisms of iron acquisition by bacterial and fungal pathogens of humans. Front. Cell. Infect. Microbiol. 3, 80 (2013)

93. Garcia, E. C., Brumbaugh, A. R. \& Mobley, H. L. Redundancy and specificity of Escherichia coli iron acquisition systems during urinary tract infection. Infect. Immun. 79, 1225-1235 (2011).

94. Watts, R. E. et al. Contribution of siderophore systems to growth and urinary tract colonization of asymptomatic bacteriuria Escherichia coli. Infect. Immun. 80, 333-344 (2012).

95. Valdebenito, M., Crumbliss, A. L., Winkelmann, G. \& Hantke, K. Environmental factors influence the production of enterobactin, salmochelin, aerobactin, and yersiniabactin in Escherichia coli strain Nissle 1917. Int. J. Med. Microbiol. 296, 513-520 (2006).

96. Chaturvedi, K. S., Hung, C. S., Crowley, J. R., Stapleton, A. E. \& Henderson, J. P. The siderophore yersiniabactin binds copper to protect pathogens during infection. Nature Chem. Biol. 8, 731-736 (2012).

97. Himpsl, S. D. et al. Proteobactin and a yersiniabactinrelated siderophore mediate iron acquisition in Proteus mirabilis. Mol. Microbiol. 78, 138-157 (2010).

98. Brumbaugh, A. R., Smith, S. N. \& Mobley, H. L. Immunization with the yersiniabactin receptor, FyuA protects against pyelonephritis in a murine model of urinary tract infection. Infect. Immun. 81, 3309-3316 (2013).

This investigation uses data from genomic, proteomic and metabolic screens to identify vaccine targets in $E$. coli., all of which are involved in iron acquisition. Vaccination with several iron receptors during experimental UTIs in mice revealed that these factors were an effective target for the development of vaccines.

99. Paterson, D. L. Resistance in Gram-negative bacteria: Enterobacteriaceae. Am. J. Infect. Control 34, S20-S28 (2006)

100. Garau, J. Other antimicrobials of interest in the era of extended-spectrum $\beta$-lactamases: fosfomycin, nitrofurantoin and tigecycline. Clin. Microbiol. Infect. 14, 198-202 (2008)

101. Pendleton, J. N., Gorman, S. P. \& Gilmore, B. F. Clinical relevance of the ESKAPE pathogens. Expert Rev. Anti Infect. Ther. 11, 297-308 (2013).

102. Gupta, K. \& Bhadelia, N. Management of urinary tract infections from multidrug-resistant organisms. Infect. Dis. Clin. North Am. 28, 49-59 (2014).

103. Bradford, P. A. Extended-spectrum $\beta$-lactamases in the 21 st century: characterization, epidemiology, and detection of this important resistance threat. Clin. Microbiol. Rev. 14, 933-951 (2001).

104. Courvalin, P. Vancomycin resistance in Gram-positive cocci. Clin. Infect. Dis. 42 (Suppl. 1), S25-S34 (2006)

105. Zhanel, G. G. et al. Ceftazidime-avibactam: a novel cephalosporin/ $\beta$-lactamase inhibitor combination. Drugs 73, 159-177 (2013).

106. Livermore, D. M. \& Mushtaq, S. Activity of biapenem (RPX2003) combined with the boronate $\beta$-lactamase inhibitor RPX7009 against carbapenem-resistant Enterobacteriaceae. J. Antimicrob. Chemother. 68 1825-1831 (2013).
107. Mushtaq, S., Woodford, N., Hope, R., Adkin, R. \& Livermore, D. M. Activity of BAL30072 alone or combined with $\beta$-lactamase inhibitors or with meropenem against carbapenem-resistant Enterobacteriaceae and non-fermenters. J. Antimicrob. Chemother. 68, 1601-1608 (2013)

108. Asadi Karam, M. R., Oloomi, M., Mahdavi, M., Habibi, M. \& Bouzari, S. Vaccination with recombinant FimH fused with flagellin enhances cellular and humoral immunity against urinary tract infection in mice. Vaccine 31, 1210-1216 (2013).

109. Langermann, S. et al. Vaccination with FimH adhesin protects cynomolgus monkeys from colonization and infection by uropathogenic Escherichia coli. J. Infect. Dis. 181, 774-778 (2000)

110. Langermann, S. et al. Prevention of mucosal Escherichia coli infection by FimH-adhesin-based systemic vaccination. Science 276, 607-611 (1997).

This pivotal study shows that blocking the interaction between the bacterial adhesin and the host receptor through vaccination can prevent UTIs in mice.

111. Roberts, J. A. et al. Antibody responses and protection from pyelonephritis following vaccination with purified Escherichia coli PapDG protein. J. Urol. 171, 1682-1685 (2004).

112. Savar, N. S. et al. In silico and in vivo studies of truncated forms of flagellin (FliC) of enteroaggregative Escherichia coli fused to FimH from uropathogenic Escherichia coli as a vaccine candidate against urinary tract infections. J. Biotechnol. 175, 31-37 (2014)

113. Li, X. et al. Use of translational fusion of the MrpH fimbrial adhesin-binding domain with the cholera toxin A2 domain, coexpressed with the cholera toxin B subunit, as an intranasal vaccine to prevent experimental urinary tract infection by Proteus mirabilis. Infect. Immun. 72, 7306-7310 (2004).

114. Sivick, K. E. \& Mobley, H. L. Waging war against uropathogenic Escherichia coli: winning back the urinary tract. Infect. Immun. 78, 568-585 (2010).

115. O'Hanley, P., Lalonde, G. \& Ji, G. Alpha-hemolysin contributes to the pathogenicity of piliated digalactoside-binding Escherichia coli in the kidney: efficacy of an $\alpha$-hemolysin vaccine in preventing renal injury in the BALB/c mouse model of pyelonephritis. Infect. Immun. 59, 1153-1161 (1991)

116. Alamuri, P., Eaton, K. A., Himpsl, S. D., Smith, S. N. $\&$ Mobley, H. L. Vaccination with proteus toxic agglutinin, a hemolysin-independent cytotoxin in vivo, protects against Proteus mirabilis urinary tract infection. Infect. Immun. 77, 632-641 (2009).

117. Alteri, C. J., Hagan, E. C., Sivick, K. E., Smith, S. N $\&$ Mobley, H. L. Mucosal immunization with iron receptor antigens protects against urinary tract infection. PLoS Pathog. 5, e1000586 (2009).

118. Nagaya, H., Satoh, H., Kubo, K. \& Maki, Y. Possible mechanism for the inhibition of gastric $\left(\mathrm{H}^{+}+\mathrm{K}^{+}\right)$adenosine triphosphatase by the proton pump inhibitor AG-1749. J. Pharmacol. Exp. Ther. 248, 799-805 (1989).

119. Sjostrom, J. E., Kuhler, T. \& Larsson, H. Basis for the selective antibacterial activity in vitro of proton pump inhibitors against Helicobacter spp. Antimicrob. Agents Chemother. 41, 1797-1801 (1997).

120. Pinkner, J. S. et al. Rationally designed small compounds inhibit pilus biogenesis in uropathogenic bacteria. Proc. Natl Acad. Sci. USA 103, 17897-17902 (2006)

121. Cusumano, C. K. et al. Treatment and prevention of urinary tract infection with orally active FimH inhibitors. Sci. Transl. Med. 3, 109ra 115 (2011).

This key work uses compounds designed to prevent the $E$. coli type 1 pilus adhesin from binding the host receptor and demonstrates that these compounds are effective at preventing UTIs in mice.

122. Greene, S. E. et al. Pilicide ec240 disrupts virulence circuits in uropathogenic E. coli. mBio 5, e02038 (2014).

This is the first paper to describe the role of pilicide in transcriptional and translational regulation in UPEC.

123. Abgottspon, D. et al. Development of an aggregation assay to screen FimH antagonists. J. Microbiol. Methods 82, 249-255 (2010).

124. Klein, T. et al. FimH antagonists for the oral treatment of urinary tract infections: from design and synthesis to in vitro and in vivo evaluation. J. Med. Chem. 53 , 8627-8641 (2010)

125. Totsika, M. et al. A FimH inhibitor prevents acute bladder infection and treats chronic cystitis caused by multidrug-resistant uropathogenic Escherichia coli ST131. J. Infect. Dis. 208, 921-928 (2013).

126. Schwartz, D. J. et al. Positively selected FimH residues enhance virulence during urinary tract infection by altering FimH conformation. Proc. Natl Acad. Sci. USA 110, 15530-15537 (2013).

This study identifies the role of key FimH residues in protein conformation and virulence.

127. Sokurenko, E. V., Chesnokova, V., Doyle, R. J. \& Hasty, D. L. Diversity of the Escherichia coli type 1 fimbrial lectin. Differential binding to mannosides and uroepithelial cells. J. Biol. Chem. 272, 17880-17886 (1997).

128. Weissman, S. J. et al. Differential stability and trade-off effects of pathoadaptive mutations in the Escherichia coli FimH adhesin. Infect. Immun. 75 3548-3555 (2007).

129. Justice, S. S., Hunstad, D. A., Cegelski, L. \& Hultgren, S. J. Morphological plasticity as a bacterial survival strategy. Nature Rev. Microbiol. 6, 162-168 (2008).

130. Horvath, D. J. Jr et al. Morphological plasticity promotes resistance to phagocyte killing of uropathogenic Escherichia coli. Microbes Infect. 13, 426-437 (2011)

131. Danese, P. N., Pratt, L. A., Dove, S. L. \& Kolter, R. The outer membrane protein, antigen 43, mediates cell-to-cell interactions within Escherichia coli biofilms Mol. Microbiol. 37, 424-432 (2000).

132. Lane, M. C., Li, X., Pearson, M. M., Simms, A. N. \& Mobley, H. L. Oxygen-limiting conditions enrich for fimbriate cells of uropathogenic Proteus mirabilis and Escherichia coli. J. Bacteriol. 191, 1382-1392 (2009).

133. Yu, H. et al. Elastase LasB of Pseudomonas aeruginosa promotes biofilm formation partly through rhamnolipid-mediated regulation. Can. J. Microbiol. 60, 227-235 (2014)

134. Diggle, S. P. et al. The galactophilic lectin, LecA, contributes to biofilm development in Pseudomonas aeruginosa. Environ. Microbiol. 8, 1095-1104 (2006)

135. Fazli, M. et al. Regulation of biofilm formation in Pseudomonas and Burkholderia species. Environ. Microbiol. 16, 1961-1981 (2014).

136. Wagner, V. E., Li, L. L., Isabella, V. M. \& Iglewski, B. H. Analysis of the hierarchy of quorum-sensing regulation in Pseudomonas aeruginosa. Anal. Bioanal. Chem. 387, 469-479 (2007).

137. Kumar, R., Chhibber, S. \& Harjai, K. Quorum sensing is necessary for the virulence of Pseudomonas aeruginosa during urinary tract infection. Kidney Int. 76, 286-292 (2009)

138. Justice, S. S., Hunstad, D. A., Seed, P. C. \& Hultgren, S. J. Filamentation by Escherichia coli subverts innate defenses during urinary tract infection. Proc. Natl Acad. Sci. USA 103 19884-19889 (2006).

139. Morgenstein, R. M. \& Rather, P. N. Role of the Umo proteins and the Rcs phosphorelay in the swarming motility of the wild type and an O-antigen (waaL) mutant of Proteus mirabilis. J. Bacteriol. 194, 669-676 (2012)

140. Walther-Rasmussen, J. \& Hoiby, N. Cefotaximases (CTX-M-ases), an expanding family of extended spectrum $\beta$-lactamases. Can. J. Microbiol. 50, 137-165 (2004).

141. Schwan, W. R. Flagella allow uropathogenic Escherichia coli ascension into murine kidneys. Int. J. Med. Microbiol. 298, 441-447 (2008).

142. Ulett, G. C. et al. Functional analysis of antigen 43 in uropathogenic Escherichia coli reveals a role in longterm persistence in the urinary tract. Infect. Immun. 75, 3233-3244 (2007)

143. Tarkkanen, A. M. et al. Fimbriation, capsulation, and iron-scavenging systems of Klebsiella strains associated with human urinary tract infection. Infect. Immun. 60, 1187-1192 (1992).

144. Podschun, R., Sievers, D., Fischer, A. \& Ullmann, U. Serotypes, hemagglutinins, siderophore synthesis, and serum resistance of Klebsiella isolates causing human urinary tract infections. J. Infect. Dis. 168, 1415-1421 (1993).

145. Dumanski, A. J., Hedelin, H., Edin-Liljegren, A., Beauchemin, D. \& McLean, R. J. Unique ability of the Proteus mirabilis capsule to enhance mineral growth in infectious urinary calculi. Infect. Immun. 62, 2998-3003 (1994). 
146. Cole, S. J., Records, A. R., Orr M. W., Linden, S. B. \& Lee, V. T. Catheter-associated urinary tract infection by Pseudomonas aeruginosa is mediated by exopolysaccharide-independent biofilms. Infect. Immun. 82, 2048-2058 (2014).

147. Gupta, P., Gupta, R. K. \& Harjai, K. Multiple virulence factors regulated by quorum sensing may help in establishment and colonisation of urinary tract by Pseudomonas aeruginosa during experimental urinary tract infection. Indian J. Med. Microbiol. 31, 29-33 (2013).

148. Hell, W., Meyer, H. G. W. \& Gatermann, S. G. Cloning of aas, a gene encoding a Staphylococcus saprophyticus surface protein with adhesive and autolytic properties. Mol. Microbiol. 29, 871-881 (1998).

149. Kline, K. A. et al. Characterization of a novel murine model of Staphylococcus saprophyticus urinary tract infection reveals roles for Ssp and Sdrl in virulence. Infect. Immun. 78, 1943-1951 (2010).
Acknowledgements

The authors apologize to researchers whose work could not be included in this Review owing to space limitations. They thank members of S.J.H.'s and M.G.C.'s laboratories, espe-

cially K. W. Dodson, for their suggestions and comments. This work was supported by the 1F32DK104516-01 grant to A.L.F.-M. and the R01-DK051406, R01-Al108749-01 and P50-DK0645400 grants from the US National Institute of Allergy and Infectious Diseases (NIAID) and US National Institute of Diabetes and Digestive and Kidney Diseases (NIDDK).

\section{Competing interests statement}

The authors declare no competing interests.

\section{SUPPLEMENTARY INFORMATION}

See online article: $\underline{\text { 1 }}$ (table)

ALL LINKS ARE ACTIVE IN THE ONLINE PDF 